MAN/HEP/2009/17

\title{
Probing SUSY CP Violation in Two-Body Stop Decays at the LHC
}

\author{
Frank F. Deppisch \\ School of Physics and Astronomy, University of Manchester, \\ Manchester M13 9PL, United Kingdom \\ Olaf Kittei 2 \\ Departamento de Física Teórica y del Cosmos and CAFPE, \\ Universidad de Granada, E-18071 Granada, Spain
}

\begin{abstract}
We study CP asymmetries in two-body decays of top squarks into neutralinos and sleptons at the LHC. These asymmetries are used to probe the CP phases possibly present in the stop and neutralino sector of the Minimal Supersymmetric Standard Model. Taking into account bounds from experimental electric dipole moment searches, we identify areas in the mSUGRA parameter space where CP asymmetries can be sizeable and discuss the feasibility of their observation at the LHC. As a result, potentially detectable CP asymmetries in stop decays at the LHC are found, motivating further detailed experimental studies for probing SUSY CP phases.
\end{abstract}

\footnotetext{
${ }^{1}$ Email: frank.deppisch@manchester.ac.uk

${ }^{2}$ Email: kittel@th.physik.uni-bonn.de
} 


\section{Introduction}

Supersymmetry (SUSY) [1] is a well motivated theory to extend the Standard Model (SM) of particle physics. SUSY models are not only favored by gauge coupling unification and naturalness considerations, but are also attractive from the cosmological point of view. For instance the lightest SUSY particle (LSP) is a good dark matter candidate if it is stable, massive and weakly interacting [2,3]. Most interestingly SUSY models provide a number of new parameters, among some having physical phases which cause manifest CP violation [4]. In the SM, the CP phase in the quark mixing matrix, which is currently confirmed in $B$ meson experiments [5, 6], cannot explain the observed baryon asymmetry of the universe [7], and additional sources of $\mathrm{CP}$ violation in models beyond the SM are required [8].

In the Minimal Supersymmetric Standard Model (MSSM) [1], a set of remaining complex parameters is obtained, after absorbing non-physical phases by redefining particle fields. The complex parameters are conventionally chosen to be the Higgsino mass parameter $\mu$, the $\mathrm{U}(1)$ and $\mathrm{SU}(3)$ gaugino mass parameters $M_{1}$ and $M_{3}$, respectively, and the trilinear scalar coupling parameters $A_{f}$ of the third generation sfermions $(f=b, t, \tau)$,

$$
\mu=|\mu| e^{i \phi_{\mu}}, \quad M_{1}=\left|M_{1}\right| e^{i \phi_{1}}, \quad M_{3}=\left|M_{3}\right| e^{i \phi_{3}}, \quad A_{f}=\left|A_{f}\right| e^{i \phi_{A_{f}}} .
$$

These phases contribute to the electric dipole moments (EDMs) of the electron, neutron and deuteron, which can be beyond their current experimental upper bounds $[5$, 9 12. However, the extent to which the EDMs can constrain the SUSY phases strongly depends on the considered model and its parameters [13 18]. Thus measurements of SUSY CP observables outside the low energy EDM sector are necessary to independently determine or constrain the phases.

For example the phases of the trilinear scalar coupling parameters $A_{f}$ have a significant impact on the MSSM Higgs sector [19]. Loop effects, dominantly mediated by third generation squarks, can generate large CP-violating scalar-pseudoscalar transitions among the neutral Higgs bosons [20]. As a result the lightest Higgs boson with a mass of order $10 \mathrm{GeV}$ or $45 \mathrm{GeV}$ [21] cannot be excluded by measurements at LEP [22]. The fundamental properties and the phenomenology of CP-violating neutral Higgs boson mixings have been investigated in detail in the literature [23].

In addition, the phases of the coupling parameters $A_{b, t, \tau}$ in the third generation sfermion sector are rather unconstrained by the EDMs [17, 18. Therefore it looks appealing to find the adequate $\mathrm{CP}$ observables in sfermion production and decays at high energy colliders like the LHC [24] or ILC [25], which are sensitive to the eventually large CP phases of $A_{b, t, \tau}$. Third generation sfermions also have a rich phenomenology due to a sizable mixing of left and right states.

The phases can drastically change SUSY particle masses, cross sections, branching ratios [26 28], and longitudinal polarizations of final fermions [29]. Although such CP-even observables are very sensitive to the CP phases (the observables can change by an order of magnitude and more), CP-odd (T-odd) observables have to be measured for a direct evidence of $\mathrm{CP}$ violation. CP-odd observables are, for example, rate asymmetries of cross sections, distributions, and partial decay widths [30]. 


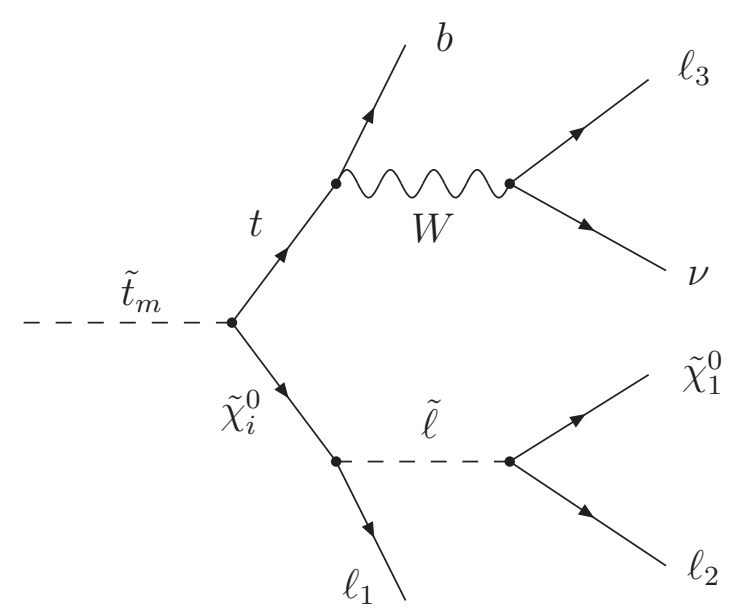

Figure 1: Schematic picture of top squark decay.

However, these observables require the presence of absorptive phases, e.g. from loops, and thus usually do not exceed the size of $10 \%$.

Larger CP asymmetries in particle decay chains can be obtained with triple products of final particle momenta [31]. They already appear at tree level due to spin correlations. Triple product asymmetries have been intensively studied in the production and decay of neutralinos [32 36] and charginos [36 38] at the ILC, also using transversely polarized beams [39]. For recent reviews, see Ref. [40].

In this paper, we study CP asymmetries in two-body decays of a stop,

$$
\tilde{t}_{m} \rightarrow t+\tilde{\chi}_{i}^{0} ; \quad m=1,2 ; \quad i=2,3,4
$$

followed by the subsequent two-body decay chains of the neutralino,

$$
\tilde{\chi}_{i}^{0} \rightarrow \ell_{1}+\tilde{\ell}_{n} ; \quad \tilde{\ell}_{n} \rightarrow \tilde{\chi}_{1}^{0}+\ell_{2} ; \quad n=L, R, \quad \ell=e, \mu,
$$

and the top quark

$$
t \rightarrow b+W ; \quad W \rightarrow \nu_{\ell}+\ell_{3} ; \quad \ell=e, \mu .
$$

See Figure 1 for a schematic picture of the entire stop decay chain. Since the stop decay, Eq. (2), is a two-body decay of a scalar particle, only the spin-spin correlations of the outgoing fermions contain a CP-sensitive part. Spin-spin correlations are terms in the amplitude squared that include the polarization vectors of both the neutralino and the top. The angular distributions of their decay products are then correlated to each other due to total angular-momentum conservation. Thus for the measurement of the top-neutralino spin-spin correlations, it is vital to include momenta from both their decays into the triple products, otherwise the CP-sensitive information in that process will be lost [41].

Triple product asymmetries have been studied in two-body decays of stops [4] and sbottoms [42], however in their rest frame only. At colliders like the LHC the particles are highly boosted, which will generally reduce the triple product asymmetries [43, 44]. We also include the production of stops at the LHC, and analyze the stop boost distributions as well as their impact on the triple product asymmetries in detail. In our numerical analysis we work within the minimal Supergravity 
Table 1: Overview of sfermion two-body decays studied in the literature to analyze $\mathrm{CP}$ phases. Two-body decays are denoted by $a \rightarrow b c$ and three-body decays by $a \rightarrow b c d$. The column 'CP Phases' lists the phases the process is sensitive to, and 'Max $\mathcal{A}_{\mathrm{CP}}$ ' gives the maximal asymmetries which have been found in the rest frame of the sfermion. Asymmetries in parentheses correspond to the decay $W \rightarrow c \bar{s}$.

\begin{tabular}{|c|c|c|c|c|c|}
\hline Process & Sub Decay 1 & Sub Decay 2 & CP Phases & Max $\mathcal{A}_{\mathrm{CP}}$ & Ref. \\
\hline$\tilde{t}_{m} \rightarrow t \tilde{\chi}_{i}^{0}$ & $t \rightarrow b W$ & $W \rightarrow \bar{\ell} \nu(c \bar{s})$ & & & \\
& $\tilde{\chi}_{i}^{0} \rightarrow \tilde{\ell} \ell$ & $\tilde{\ell} \rightarrow \ell \tilde{\chi}_{1}^{0}$ & $\phi_{A_{t}}, \phi_{\mu}, \phi_{M_{1}}$ & $40 \%$ & {$[41]$} \\
\hline$\tilde{t}_{m} \rightarrow t \tilde{\chi}_{i}^{0}$ & $t \rightarrow b W$ & & & & \\
& $\tilde{\chi}_{i}^{0} \rightarrow \ell \bar{\ell} \tilde{\chi}_{1}^{0}$ & & $\phi_{A_{t}}, \phi_{\mu}, \phi_{M_{1}}$ & $15 \%$ & {$[43]$} \\
\hline$\tilde{b}_{m} \rightarrow t \tilde{\chi}_{j}^{ \pm}$ & $t \rightarrow b W$ & $W \rightarrow \bar{\ell} \nu(c \bar{s})$ & & & \\
& $\tilde{\chi}_{j}^{ \pm} \rightarrow \ell \tilde{\nu}$ & & & $15(40) \%$ & \\
& $\tilde{\chi}_{j}^{ \pm} \rightarrow \tilde{\ell} \nu$ & $\tilde{\ell} \rightarrow \ell \tilde{\chi}_{1}^{0}$ & & $5(10) \%$ & \\
& $\tilde{\chi}_{j}^{ \pm} \rightarrow W \tilde{\chi}_{1}^{0}$ & & & $2(5) \%$ & \\
& $\tilde{\chi}_{j}^{ \pm} \rightarrow W \tilde{\chi}_{1}^{0}$ & $W \rightarrow \ell \bar{\nu}(\bar{c} s)$ & $\phi_{A_{b}}, \phi_{\mu}$ & $2(5) \%$ & {$[42]$} \\
\hline$\tilde{f} \rightarrow f \tilde{\chi}_{i}^{0}$ & $\tilde{\chi}_{i}^{0} \rightarrow Z \tilde{\chi}_{1}^{0}$ & $Z \rightarrow \ell \bar{\ell}$ & & $5 \%$ & \\
& & $Z \rightarrow c \bar{c}$ & & $15 \%$ & \\
& & $Z \rightarrow b \bar{b}$ & $\phi_{\mu}, \phi_{M_{1}}$ & $20 \%$ & {$[33]$} \\
\hline$\tilde{q} \rightarrow q \tilde{\chi}_{i}^{0}$ & $\tilde{\chi}_{i}^{0} \rightarrow \ell \bar{\ell} \tilde{\chi}_{1}^{0}$ & & $\phi_{\mu}, \phi_{M_{1}}$ & $\mathrm{n} / \mathrm{a}$ & {$[44]$} \\
\hline$\tilde{\ell} \rightarrow \ell \tilde{\chi}_{i}^{0}$ & $\tilde{\chi}_{i}^{0} \rightarrow \ell \bar{\ell} \tilde{\chi}_{1}^{0}$ & & $\phi_{\mu}, \phi_{M_{1}}$ & $15 \%$ & {$[47]$} \\
\hline
\end{tabular}

(mSUGRA) framework of SUSY breaking [1, and explicitly add CP phases for the parameters $A_{t}, M_{1}, \mu$ at the electroweak scale, fully taking into account the implications on the EDMs. In addition, we calculate the amplitude squared for the entire stop decay in the spin-density matrix formalism [45]. The compact form of the amplitude squared allows us to identify the optimal $\mathrm{CP}$ observables for squark decays. For a recent study of $\mathrm{CP}$-odd observables in stop decays, including their production, but for neutralino three-body decays, see Ref. [43. A summary of the literature in sfermion two-body decays is shown in Table 1. Three-body decays of stops have been studied in Ref. [46].

The paper is organized as follows. In Section 2, we identify the CP-sensitive parts in the amplitude squared, classify the CP asymmetries in top squark decays and discuss their dependence on the complex stop-top-neutralino couplings. Section 3 contains a discussion of the experimental EDM constraints on the SUSY CP 
phases and asymmetries. For the LHC, we discuss in Section 4 the SUSY parameter dependence of stop production, boost distribution and branching ratios, and give lower bounds on the required luminosities to observe the asymmetries over their statistical fluctuations. Section 4 also includes a discussion of angular and volume distributions of the triple products. We summarize and give our conclusions in Section 5. In the Appendix, the stop and neutralino Lagrangians with complex couplings are reviewed, we give the phase space and calculate the stop decay amplitudes in the spin-density matrix formalism. We also show that absorptive phases can be eliminated from the T-odd asymmetries to obtain true CP asymmetries.

\section{CP Asymmetries in Top Squark Decays}

In this Section, we identify the CP-sensitive parts in the amplitude squared of the two-body decay of the top squark, see Eqs. (2)-(41) and Figure 1. In order to probe these parts, we systematically classify $\mathrm{T}$ - and CP-odd asymmetries. These can be defined from various triple or epsilon products of the different final state particle three- or four-momenta $p_{t}, p_{b}, p_{\ell_{1}}, p_{\ell_{2}}, p_{\ell_{3}}$, respectively. Explicit expressions for the squared amplitude, Lagrangians, couplings, and phase space elements are summarized in the Appendix.

\section{$2.1 \quad$ T-odd Products}

The amplitude squared for the stop decay chain, see Figure 1, can be decomposed into contributions from the top spin correlations, the neutralino spin correlations, the top-neutralino spin-spin correlations, and an unpolarized part, see Eq. (E.62). The amplitude squared is a sum of terms which are even and one term which is odd under a $\mathrm{T}$ transformation. Assuming CPT invariance, $\mathrm{CP}$ asymmetries are proportional to the T-odd term in the amplitude squared. Such a term originates only from the spin-spin correlations. It is given by the last summand in Eq. (E.67), which is proportional to

$$
|T|^{2} \supset \operatorname{Im}\left\{a_{m i}^{\tilde{t}}\left(b_{m i}^{\tilde{t}}\right)^{*}\right\}\left[p_{\tilde{t}}, p_{t}, p_{\ell_{3}}, p_{\ell_{1}}\right], \quad m=1,2, \quad i=1, \ldots, 4 .
$$

The left and right couplings $a_{m i}^{\tilde{t}}$ and $b_{m i}^{\tilde{t}}$ are defined through the $\tilde{t}_{m}-t-\tilde{\chi}_{i}^{0}$ Lagrangian [41,

$$
\mathscr{L}_{t \tilde{t} \tilde{\chi}^{0}}=g \bar{t}\left(a_{m i}^{\tilde{t}} P_{R}+b_{m i}^{\tilde{t}} P_{L}\right) \tilde{\chi}_{i}^{0} \tilde{t}_{m}+\text { h.c. }
$$

which we give explicitly in Appendix C. These couplings depend on the mixing in the stop and neutralino sector, and thus also on the CP phases $\phi_{A_{t}}, \phi_{\mu}$ and $\phi_{M_{1}}$. The imaginary part of the coupling product, $\operatorname{Im}\left\{a_{m i}^{\tilde{t}}\left(b_{m i}^{\tilde{t}}\right)^{*}\right\}$, in Eq. (5) is multiplied by a T-odd epsilon product, for which we have used the short hand notation

$$
\left[p_{1}, p_{2}, p_{3}, p_{4}\right] \equiv \varepsilon_{\mu \nu \alpha \beta} p_{1}^{\mu} p_{2}^{\nu} p_{3}^{\alpha} p_{4}^{\beta},
$$

with the convention $\varepsilon_{0123}=1$. Since each of the spatial components of the fourmomenta changes sign under a naive time transformation, $t \rightarrow-t$, this product is T-odd. 
In order to construct asymmetries that probe this CP-sensitive part, we consider an epsilon product of four four-momenta $p_{i}$

$$
\mathcal{E}=\left[p_{1}, p_{2}, p_{3}, p_{4}\right]
$$

The $p_{i}$ can in principle be any four linearly independent momenta in the two-body decay-chain of the top squark, $p_{\tilde{t}}, p_{t}, p_{b}, p_{\ell_{1}}, p_{\ell_{2}}, p_{\ell_{3}}$, see Figure1. Note however, that momenta from both the decay products of neutralino and the top have to be included in the epsilon product to obtain non-vanishing asymmetries [41]. Otherwise the topneutralino spin-spin correlations are lost. Only they have CP-sensitive terms, since the stop decay, Eq. (2), is a two-body decay of a scalar particle3.

\section{$2.2 \quad$ T-odd Asymmetries}

For a given T-odd product $\mathcal{E}$, Eq. (88), we can now define the T-odd asymmetry of the partial stop decay width $\Gamma$ [41],

$$
\mathcal{A}=\frac{\Gamma(\mathcal{E}>0)-\Gamma(\mathcal{E}<0)}{\Gamma(\mathcal{E}>0)+\Gamma(\mathcal{E}<0)}=\frac{\int \operatorname{Sign}[\mathcal{E}]|T|^{2} d \operatorname{Lips}}{\int|T|^{2} d \operatorname{Lips}},
$$

with the amplitude squared $|T|^{2}$, and the Lorentz invariant phase-space element $d$ Lips, such that $\frac{1}{2 m_{\tilde{t}}} \int|T|^{2} d$ Lips $=\Gamma$. The T-odd asymmetry is also CP-odd, if absorptive phases (from higher order final-state interactions or finite-width effects) can be neglected [31]. In Appendix G, we also construct a $\mathrm{CP}$ asymmetry, where those contributions are eliminated.

In general the largest asymmetries are obtained by using an epsilon product which matches the kinematic dependence of the CP-sensitive terms in the amplitude squared. In the literature, this technique is sometimes referred to optimal observables [48]. Thus the largest asymmetry is obtained from the epsilon product

$$
\mathcal{E}=\left[p_{\tilde{t}}, p_{t}, p_{\ell_{3}}, p_{\ell_{1}}\right]
$$

Other combinations of momenta in general lead to smaller asymmetries, see Ref. [41].

Triple products of three spatial momenta can also be used to define asymmetries [31]. In the stop rest frame, $p_{\tilde{t}}^{\mu}=\left(m_{\tilde{t}}, \mathbf{0}\right)$, the epsilon product reads [41]

$$
\left[p_{\tilde{t}}, p_{t}, p_{\ell_{3}}, p_{\ell_{1}}\right]=m_{\tilde{t}} \mathbf{p}_{t} \cdot\left(\mathbf{p}_{\ell_{3}} \times \mathbf{p}_{\ell_{1}}\right) \equiv m_{\tilde{t}} \mathcal{T}
$$

That triple product has been found to give the largest asymmetries in the stop rest frame, and other combinations of momenta for $\mathcal{T}$ lead to smaller asymmetries [41]. Note that the asymmetries of an epsilon product $\mathcal{E}$ are by construction Lorentz

\footnotetext{
${ }^{3}$ If for example momenta from the top decay are not taken into account, only CP asymmetries can be obtained, which are sensitive to the CP phases $\phi_{\mu}$ and $\phi_{M_{1}}$ alone, which enter solely from the neutralino decay. Still in that case, a three-body neutralino decay is required 43, 44, 47, or a two-body decay chain with an intermediate $Z$-boson [33. However, the sfermion then merely serves as a production channel for neutralinos, whose CP properties are studied through their subsequent decays [33, 43, 44, 47]. The asymmetries are then only of the order of $10 \%$, which is typical for neutralino three-body decays [33, 35, 43, 44, 47], and also chargino three-body decays [38].
} 
Table 2: mSUGRA benchmark scenario, extended with SUSY CP phases at the electroweak scale.

\begin{tabular}{ccccccc}
\hline$m_{0}$ & $m_{1 / 2}$ & $A_{0}$ & $\tan \beta$ & $\phi_{A_{t}}$ & $\phi_{\mu}$ & $\phi_{M_{1}}$ \\
\hline $70 \mathrm{GeV}$ & $270 \mathrm{GeV}$ & $500 \mathrm{GeV}$ & 5 & $\frac{1}{5} \pi$ & 0 & 0 \\
\hline
\end{tabular}

Table 3: SUSY parameters and masses at the electroweak scale for the benchmark scenario of Table $2(\ell=e, \mu)$.

\begin{tabular}{|c|c|c|c|}
\hline$M_{1}=110 \mathrm{GeV}$ & $M_{2}=206 \mathrm{GeV}$ & $\mu=344 \mathrm{GeV}$ & $\left|A_{t}\right|=329 \mathrm{GeV}$ \\
\hline \hline$m_{\tilde{t}_{1}}=472 \mathrm{GeV}$ & $m_{\tilde{t}_{2}}=612 \mathrm{GeV}$ & $m_{\tilde{b}_{1}}=560 \mathrm{GeV}$ & $m_{\tilde{b}_{2}}=581 \mathrm{GeV}$ \\
\hline$m_{\chi_{1}^{0}}=102 \mathrm{GeV}$ & $m_{\chi_{2}^{0}}=189 \mathrm{GeV}$ & $m_{\chi_{3}^{0}}=350 \mathrm{GeV}$ & $m_{\chi_{4}^{0}}=376 \mathrm{GeV}$ \\
\hline$m_{\chi_{1}^{ \pm}}=187 \mathrm{GeV}$ & $m_{\chi_{2}^{ \pm}}=376 \mathrm{GeV}$ & $m_{\tilde{\ell}_{R}}=130 \mathrm{GeV}$ & $m_{\tilde{\ell}_{L}}=201 \mathrm{GeV}$ \\
\hline
\end{tabular}

invariant whereas those constructed with a triple product $\mathcal{T}$ are not [44]. The triple product asymmetries will therefore depend on the stop boost, $\beta_{\tilde{t}}=\left|\mathbf{p}_{\tilde{t}}\right| / E_{\tilde{t}}$, and are generally reduced if not evaluated in the stop rest frame [49]. We will discuss in detail the impact of the stop boost on the asymmetries at the LHC in Section 4.

\subsection{Phase Dependence}

In order to analyze the phase dependence of the asymmetry $\mathcal{A}$, Eq. (9), we insert the explicit form of the amplitude squared in the spin-density formalism. As shown in Appendix E. we obtain

$$
\mathcal{A}=2 \eta \frac{\int \operatorname{Sign}(\mathcal{E})\left(p_{b} \cdot p_{\nu_{\ell}}\right)\left[p_{\tilde{t}}, p_{t}, p_{\ell_{3}}, p_{\ell_{1}}\right] d \operatorname{Lips}}{\left(m_{\chi_{i}^{0}}^{2}-m_{\tilde{\ell}}^{2}\right) \int\left(p_{t} \cdot p_{\ell_{3}}\right)\left(p_{b} \cdot p_{\nu_{\ell}}\right) d \operatorname{Lips}},
$$

with the coupling function

$$
\eta=\frac{\operatorname{Im}\left\{a_{m i}^{\tilde{t}}\left(b_{m i}^{\tilde{t}}\right)^{*}\right\}}{\frac{1}{2}\left(\left|a_{m i}^{\tilde{t}}\right|^{2}+\left|b_{m i}^{\tilde{t}}\right|^{2}\right) \frac{m_{\tilde{t}}^{2}-m_{\chi_{i}^{0}}^{2}-m_{t}^{2}}{2 m_{\chi_{i}^{0}} m_{t}}-\operatorname{Re}\left\{a_{m i}^{\tilde{t}}\left(b_{m i}^{\tilde{t}}\right)^{*}\right\}} .
$$

Thus the asymmetry can be separated into a kinematical part, and the effective coupling factor $\eta$, that is approximately independent of the particle masses, but governs the complete phase dependence of the stop-top-neutralino couplings.

To discuss the phase dependence of $\eta$ (and $\mathcal{A}$ ), we choose the mSUGRA framework of SUSY breaking [1]. We extend it by adding the CP phases $\phi_{A_{t}}, \phi_{\mu}$ and $\phi_{M_{1}}$ at the electroweak scale. We define a benchmark scenario in Table 2, and give the 

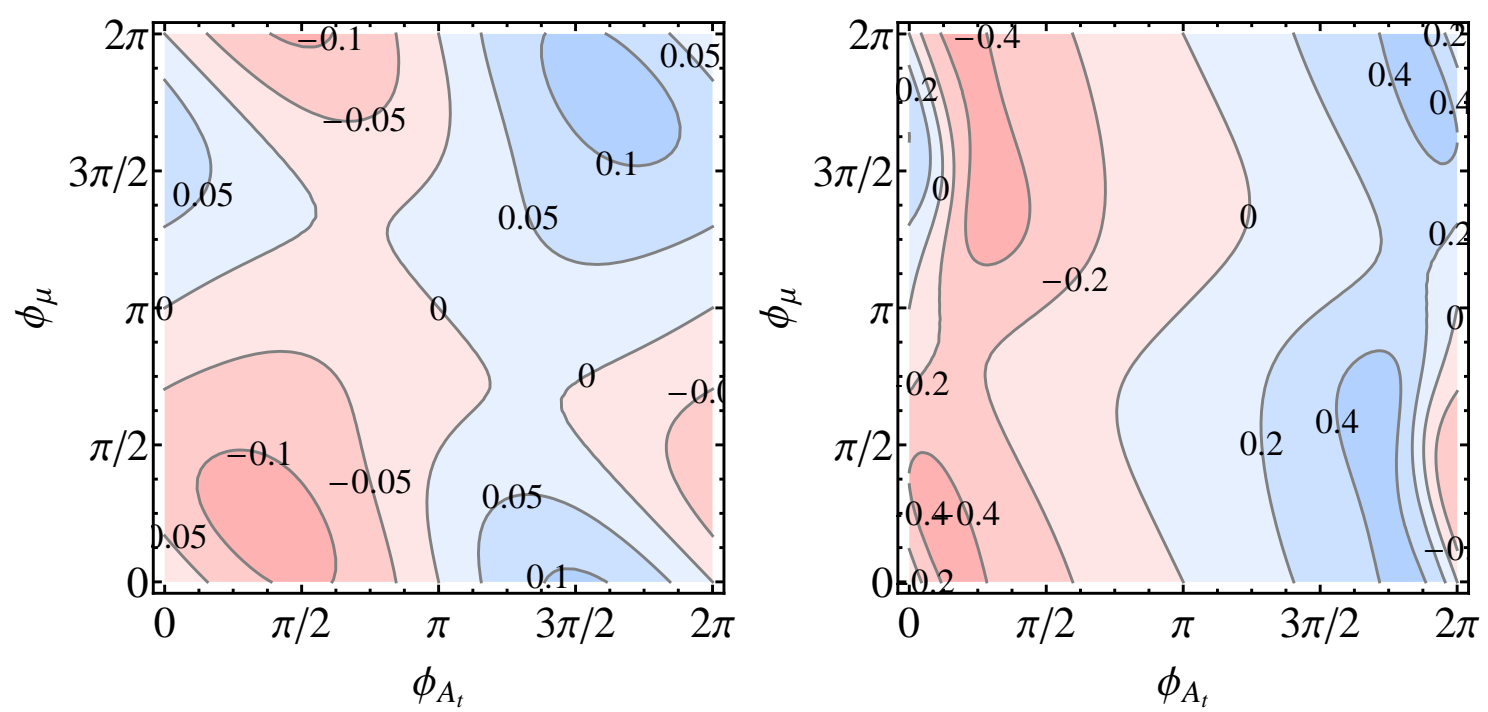

Figure 2: Approximated CP-odd coupling factor $\operatorname{Im}\left\{a_{12}^{\tilde{t}}\left(b_{12}^{\tilde{t}}\right)^{*}\right\}$, Eq. (14), (left), and complete effective coupling factor $\eta$, Eq. (13), (right) for the decay $\tilde{t}_{1} \rightarrow t \tilde{\chi}_{2}^{0}$, as a function of the $\mathrm{CP}$ phases $\phi_{A_{t}}$ and $\phi_{\mu}$. All other phases are set to zero. The mSUGRA parameters are given in Table 2 .

other low energy parameters and masses in Table 3. The scenario is chosen to approximately maximize the signals at the LHC, while still respecting current bounds from electric dipole moments, see the detailed discussion in Section 3 .

Within mSUGRA, the lightest neutralino is essentially a bino whereas the second lightest neutralino is a wino. For the stop decay via the second lightest neutralino, $\tilde{t}_{1} \rightarrow t \tilde{\chi}_{2}^{0}$, the asymmetries will primarily depend on $\phi_{A_{t}}$ and $\phi_{\mu}$, through the influence of stop mixing. We expect a weak dependence on $\phi_{M_{1}}$, since $\tilde{\chi}_{2}^{0}$ has a negligible bino component. In this limit of the neutralino mixing matrix [18], valid for a large part of the mSUGRA parameter space, the phase dependence of the CP-odd coupling factor $\operatorname{Im}\left\{a_{m i}^{\tilde{t}}\left(b_{m i}^{\tilde{t}}\right)^{*}\right\}$ for the decay $\tilde{t}_{1} \rightarrow t \tilde{\chi}_{2}^{0}$ can be approximated as

$$
\begin{aligned}
\operatorname{Im}\left\{a_{12}^{\tilde{t}}\left(b_{12}^{\tilde{t}}\right)^{*}\right\} \propto & \frac{\cos ^{2} \theta_{\tilde{t}}}{\tan \beta} \frac{m_{t}|\mu|}{|\mu|^{2}-M_{2}^{2}} \sin \left(\phi_{\mu}\right) \\
& +\frac{\sin 2 \theta_{\tilde{t}}}{2}\left|\frac{m_{t} M_{2}}{|\mu|^{2}-M_{2}^{2}}\right|^{2} \sin \left(\phi_{A_{t}}\right) \\
& +\frac{\sin 2 \theta_{\tilde{t}}}{\tan \beta}\left|\frac{m_{t} \sqrt{|\mu| M_{2}}}{|\mu|^{2}-M_{2}^{2}}\right|^{2} \sin \left(\phi_{A_{t}}+\phi_{\mu}\right) \\
& +\frac{\sin 2 \theta_{\tilde{t}}}{\tan ^{2} \beta}\left|\frac{m_{t}|\mu|}{|\mu|^{2}-M_{2}^{2}}\right|^{2} \sin \left(\phi_{A_{t}}+2 \phi_{\mu}\right)
\end{aligned}
$$

with the stop mixing angle $\theta_{\tilde{t}}$, defined in Eq. (A.8).

In Figure 2, we show the phase dependence of $\operatorname{Im}\left\{a_{12}^{\tilde{t}}\left(b_{12}^{\tilde{t}}\right)^{*}\right\}$ in its approximation, Eq. (14), for the mSUGRA scenario of Table 2, For comparison we also show the coupling factor $\eta$, Eq. (13), without approximations. The deviations are due to 

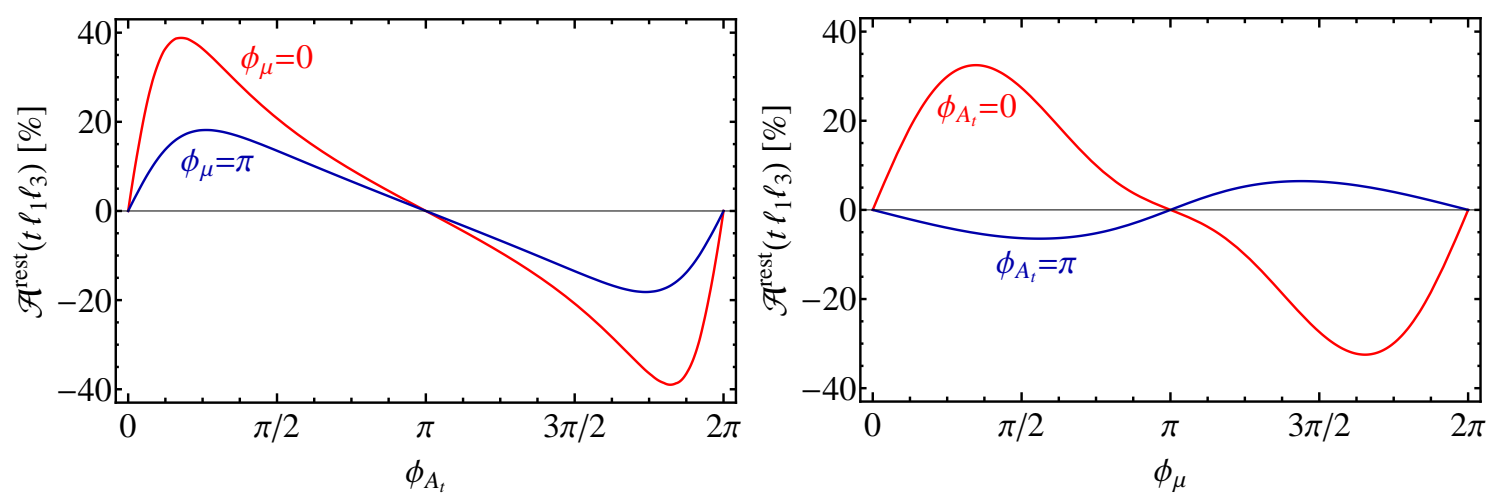

Figure 3: Asymmetry $\mathcal{A}\left(t \ell_{1} \ell_{3}\right)$, Eq. (9), as a function of the CP phase $\phi_{A_{t}}$ with $\phi_{\mu}=0, \pi$ (left), and as a function of $\phi_{\mu}$ with $\phi_{A_{t}}=0, \pi$ (right), for the stop decay $\tilde{t}_{1} \rightarrow t \tilde{\chi}_{2}^{0}$ and $\tilde{\chi}_{2}^{0} \rightarrow \ell_{1} \tilde{\ell}_{R}$, see Figure 1, in the stop rest frame. The mSUGRA parameters are given in Table 2 .

CP-even terms in the denominator of $\eta$. The CP-even parts are generally functions of the cosine of the phases. Figure 2 illustrates that such terms have an important impact on the phase dependence of the CP asymmetries.

We further illustrate this effect on the asymmetry $\mathcal{A}(12)$ for the optimal epsilon product $\mathcal{E}$ (10), for $p p \rightarrow \tilde{t}_{1} \tilde{t}_{1}^{*}$ production and the subsequent decays $\tilde{t}_{1} \rightarrow t \tilde{\chi}_{2}^{0}$ and $\tilde{\chi}_{2}^{0} \rightarrow \ell \tilde{\ell}_{R}$ via the second lightest neutralino and a right slepton. In the stop rest frame, this asymmetry is equivalent to the triple product asymmetry with $\mathcal{T}=$ $\mathbf{p}_{t} \cdot\left(\mathbf{p}_{\ell_{3}} \times \mathbf{p}_{\ell_{1}}\right)$, as given in Eq. (11). We use the short hand notation $\mathcal{A}\left(t \ell_{1} \ell_{3}\right)$, and similarly for other asymmetries to indicate the momenta used for the triple product.

In Figure 3, we show $\mathcal{A}\left(t \ell_{1} \ell_{3}\right)$ as a function of $\phi_{A_{t}}$ (left panel), and $\phi_{\mu}$ (right panel). The dependence on $\phi_{M_{1}}$ is numerically small as expected. We clearly see that the maxima of $\mathcal{A}\left(t \ell_{1} \ell_{3}\right) \approx \pm 40 \%$ are not necessarily obtained for maximal $\mathrm{CP}$ phases $\phi_{\mu}, \phi_{A_{t}}=\pi / 2,3 / 2 \pi$. The reason is that the phase dependence of $\mathcal{A}\left(t \ell_{1} \ell_{3}\right)$ is almost governed by the coupling factor $\eta$, see Figure2, right panel. Since the CP-odd (CP-even) numerator (denominator) of $\eta$ has a sine-like (cosine-like) dependence on the phases, the maxima of $\eta$, and thus in turn of $\mathcal{A}\left(t \ell_{1} \ell_{3}\right)$, are shifted away from $\phi_{A_{t}}$, $\phi_{\mu}=\pi / 2,3 / 2 \pi$ in Figure 3. Thus the asymmetry can be sizable for smaller values of the phases, which is favored by EDM constraints. For example, the asymmetry has a maximum at $\phi_{\mu} \approx 3 / 8 \pi$ for $\phi_{A_{t}}=0$, and for $\phi_{\mu}=0$ the asymmetry is maximal at $\phi_{A_{t}} \approx 1 / 5 \pi$. The positions of the maxima will remain when including the effects of the stop boost, as we will discuss in Section 4.1. This motivates the choice $\phi_{A_{t}}=1 / 5 \pi, \phi_{\mu}=0$ at the weak scale in our benchmark scenario (Table 2).

\section{Bounds from Electric Dipole Moments}

The CP-violating phases in SUSY models are constrained by limits on the T-odd electric dipole moments (EDMs) [17]. The current experimental bounds on the 


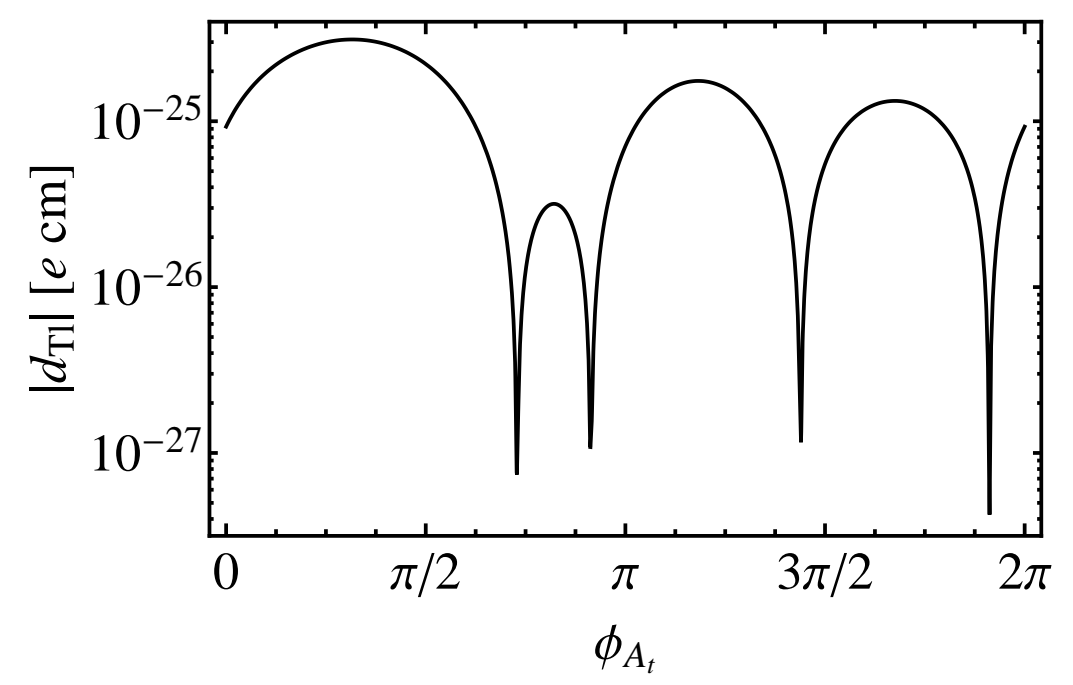

Figure 4: Electric dipole moment of Thallium as a function of $\phi_{A_{t}}$ with $\phi_{A_{b}}=$ $40 / 180 \pi, \phi_{A_{\tau}}=1 / 180 \pi, \phi_{M_{3}}=10 / 180 \pi$ and $\phi_{M_{1}}=\phi_{\mu}=0$. The mSUGRA parameters are given in Table 2 .

EDMs of Thallium [9], neutron [10], and Mercury [11] are, respectively,

$$
\begin{aligned}
\left|d_{\mathrm{Tl}}\right| & =9 \cdot 10^{-25} \mathrm{ecm}, \\
\left|d_{\mathrm{n}}\right| & =3 \cdot 10^{-26} \mathrm{ecm}, \\
\left|d_{\mathrm{Hg}}\right| & =3 \cdot 10^{-29} \mathrm{e} \mathrm{cm} .
\end{aligned}
$$

A planned experiment for measuring the deuteron EDM aims at a sensitivity of [12]

$$
\left|d_{\mathrm{d}}\right|=1-3 \cdot 10^{-27} \text { e cm. }
$$

These limits generically restrict the CP phases to be smaller than $\pi / 10$, in particular the phase $\phi_{\mu}$ [15, 18]. However, cancellations between different contributions to the EDMs can allow for larger CP phases of the order of $\pi / 4$ [17].

As an example of such cancellations, Figure 4 shows the EDM of Thallium as a function of $\phi_{A_{t}}$, for $\phi_{A_{b}}=40 / 180 \pi, \phi_{A_{\tau}}=1 / 180 \pi, \phi_{M_{3}}=10 / 180 \pi$ and $\phi_{M_{1}}=\phi_{\mu}=0$. The other mSUGRA parameters are given in Table 2. At four particular values of $\phi_{A_{t}}$, seen by distinct dips in the plot, cancellations reduce $d_{\mathrm{Tl}}$ by more than two orders of magnitude.

We now randomly scatter the phases $\phi_{A_{b}}, \phi_{A_{\tau}}, \phi_{M_{3}}, \phi_{M_{1}}$ and $\phi_{A_{t}}$, setting the most constrained phase $\phi_{\mu}=0$. For each scatter point, we calculate the maximal excess of the EDMs over the corresponding experimental limit with the software package CPsuperH [50]. For the set of scatter points, we then show the line of the minima of all the excesses obtained as a function of $\phi_{A_{t}}$ in Figure 5. For $\phi_{A_{t}}<\pi$ the minima are well below the experimental limit, and for $\phi_{A_{t}}>\pi$ only slightly above. So even large values of $\phi_{A_{t}}$ are not yet excluded by the current EDM limits. Note that a more exhaustive scan to find narrow cancellation gaps would probably make even smaller experimental EDM excesses possible. For comparison, we show 


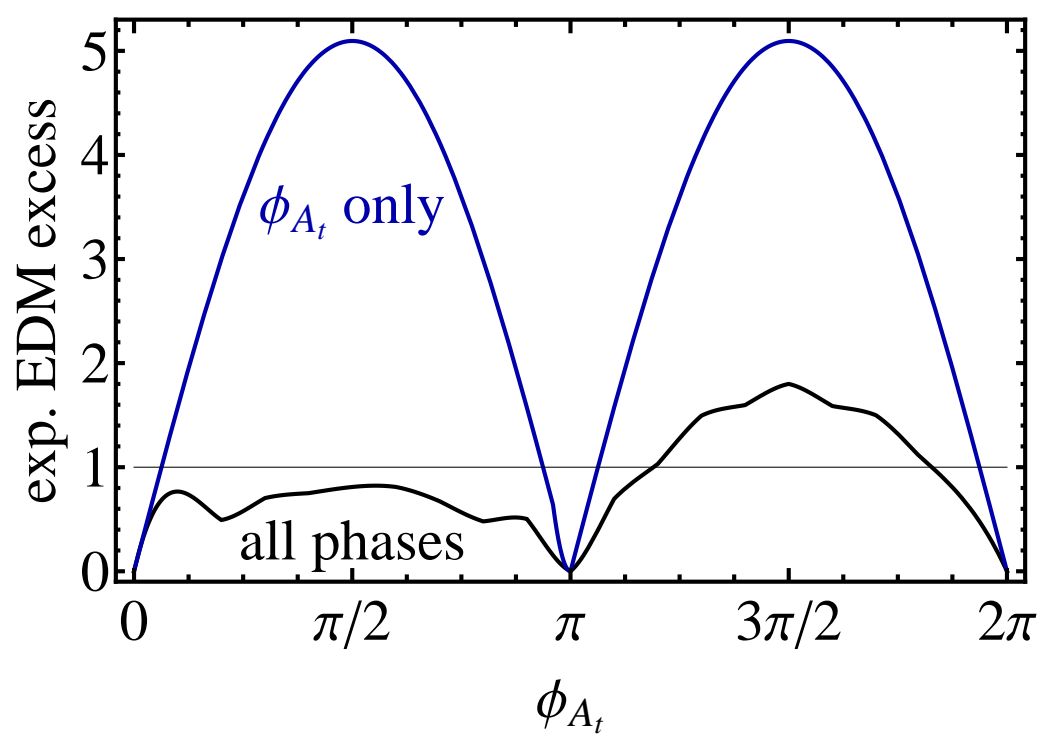

Figure 5: Experimental excess in electric dipole moments as a function of $\phi_{A_{t}}$ for $\phi_{\mu}=0$. The experimental excess is defined as the $\operatorname{maximum} \max _{i}\left|d_{i}\left(\phi_{A_{t}}, \ldots\right)\right| /\left|d_{i}^{\text {exp }}\right|$, $i=\mathrm{Hg}, \mathrm{n}, \mathrm{Tl}$, over all current experimental limits. The two curves correspond to the case where: all phases except $\phi_{A_{t}}$ are set to zero (' $\phi_{A_{t}}$ only'), and the phases $\phi_{A_{t}}$, $\phi_{A_{b}}, \phi_{A_{\tau}}, \phi_{M_{1}}$ and $\phi_{M_{3}}$ are randomly scattered ('all phases'). For a given value of $\phi_{A_{t}}$, the minimal excess is plotted. The mSUGRA parameters are given in Table 2 .

in Figure 5 also the experimental excess in the case that all phases except $\phi_{A_{t}}$ are set to zero. This demonstrates that a conspiracy among the phases can considerably reduce the predicted electric dipole moments.

We conclude that due to cancellations among different contributions to the EDMs only isolated points in the CP phase space can give large CP signals at the LHC. It is important to search for these signals, since the cancellations could be a consequence of a deeper model that correlates the phases. For instance, the existing EDM bounds can also be fulfilled by including lepton flavor violating couplings in the slepton sector [16]. This is important when considering for example SUSY Seesaw models, where $\mathrm{CP}$ violation in the slepton sector is connected to the neutrino sector and Leptogenesis [51,52].

The interrelation between the EDMs and the SUSY CP phases show that CP observables have to be determined outside the low energy sector, for example by measurements at the LHC and ILC. Asymmetries of triple products generally depend in a different way on the SUSY parameters than the EDMs, in particular on the CP phases, giving rise to a potential synergy between experiments at the low and the high energy scale. Depending on the experimental results, the EDM bounds could be either verified, or the CP-violating sectors of the model have to be modified. CP-odd observables like triple product asymmetries would be the ideal tool for such independent measurements. 

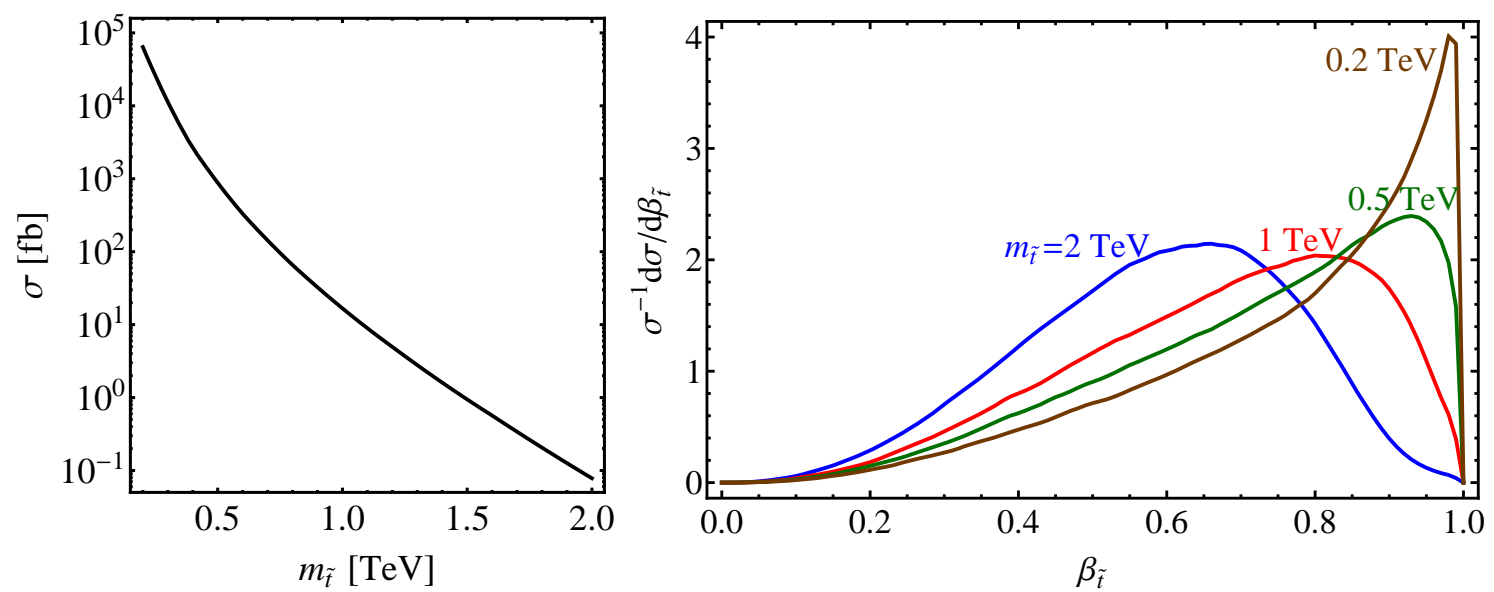

Figure 6: Total stop pair production cross section $\sigma\left(p p \rightarrow \tilde{t}^{*}\right)$ at the LHC as a function of the stop mass (left). Normalized stop pair production distribution $\frac{1}{\sigma} \frac{d \sigma}{d \beta_{\tilde{t}}}$ with respect to the stop boost factor $\beta_{\tilde{t}}$ (right). The leading order cross sections have been calculated at $\sqrt{s_{p p}}=14 \mathrm{TeV}$ using MadGraph [54].

\section{CP Asymmetries at the LHC}

At the LHC, squarks are produced with a distinct boost distribution which reduces the triple product asymmetries in the laboratory (lab) frame. In contrast to the epsilon product asymmetries they are not Lorentz invariant. If the stop rest frame cannot be reconstructed, the triple product asymmetries have to be folded with the stop boost distribution.

In this Section we will discuss the dependence of triple product asymmetries on the stop boost. To this end we first review the production cross section of stops pairs, show their boost distribution, and explain how the triple product asymmetries in the lab frame are obtained. After defining their theoretical statistical significance, we study the asymmetries, stop and neutralino branching ratios, and the significances in the mSUGRA parameter space.

\subsection{Stop Pair Production and Stop Boost Distribution}

The production of stop pairs at the LHC [53]

$$
p+p \rightarrow \tilde{t}_{m}+\tilde{t}_{m}^{*}, \quad m=1,2,
$$

dominantly proceeds via gluon fusion. As a result, the leading order cross section $\sigma\left(p p \rightarrow \tilde{t}_{m} \tilde{t}_{m}^{*}\right)$ is independent of any other SUSY model parameters than the stop mass [53]. The strong dependence can be seen in Figure 6 (left), where the cross section drops by six orders of magnitude with an increase of the stop mass from $0.2 \mathrm{TeV}$ to $2 \mathrm{TeV}$. For the LHC center-of-mass energy $\sqrt{s_{p p}}=14 \mathrm{TeV}$, we have calculated the hadronic cross section at leading order with the software package MadGraph [54]. Next to leading order (NLO) calculations for stop production have 

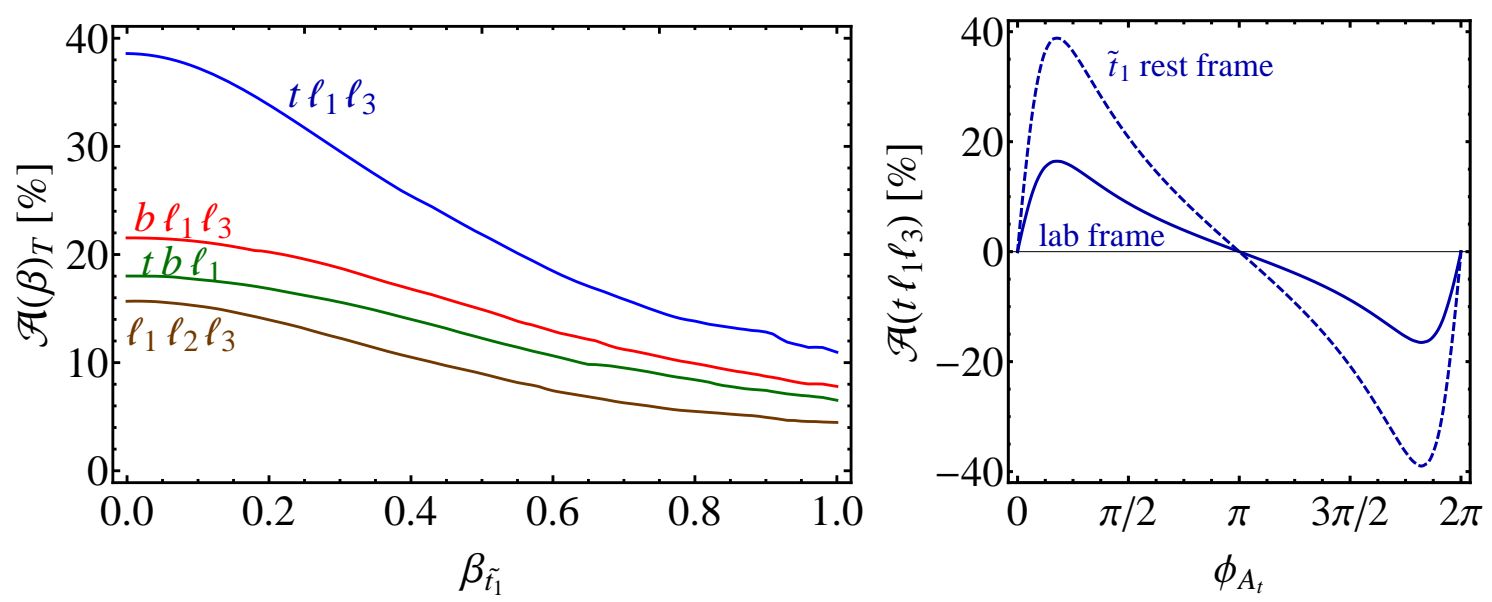

Figure 7: Asymmetries $\mathcal{A}\left(t \ell_{1} \ell_{3}\right), \mathcal{A}\left(b \ell_{1} \ell_{3}\right), \mathcal{A}\left(t b \ell_{1}\right), \mathcal{A}\left(\ell_{1} \ell_{2} \ell_{3}\right)$, see Eq. (9), for stop decay $\left(\tilde{t}_{1} \rightarrow t \tilde{\chi}_{2}^{0}, \tilde{\chi}_{2}^{0} \rightarrow \ell_{1} \tilde{\ell}_{R}\right.$, see Figure 11) as a function of the stop boost $\beta_{\tilde{t}_{1}}$ (left). Asymmetry $\mathcal{A}\left(t \ell_{1} \ell_{3}\right)$ in the stop rest frame and the laboratory frame as a function of $\phi_{A_{t}}$ with $\phi_{\mu}=\phi_{M_{1}}=0$ (right). The mSUGRA parameters are given in Table 2 .

been performed in Ref. [53], and soft gluon resummation for light-flavored squarkantisquark pairs at next to NLO has been performed in Ref. [55. Mixed stop pairs can only be generated at order $\alpha_{s}^{4}$ with a strongly suppressed rate [53].

The produced stops have a distinct distribution in their boost

$$
\beta_{\tilde{t}}=\frac{\left|\mathbf{p}_{\tilde{t}}\right|}{E_{\tilde{t}}},
$$

along the direction of their momenta. In Figure 6 (right), we show the normalized boost distribution for several values for the stop mass. In our benchmark scenario (Table 2), the lightest stop has a mass of $m_{\tilde{t}_{1}}=470 \mathrm{GeV}$ and its' boost distribution peaks at $\beta_{\tilde{t}_{1}} \approx 0.9$. Thus the decaying stop is highly boosted in the laboratory frame, which reduces the triple product asymmetries as shown in the next Section.

\subsection{Triple Product Asymmetries in the Laboratory Frame}

The asymmetries $\mathcal{A}$, Eq. (9), which are based on epsilon products $\mathcal{E}=\left[p_{1}, p_{2}, p_{3}, p_{4}\right]$, Eq. (8), are by construction Lorentz invariant. The corresponding asymmetries with triple products depend on the stop boost. In Figure 7 (left), we show the boost dependence of asymmetries for various triple product combinations $\mathcal{T}=\left(t \ell_{1} \ell_{3}\right)$, $\left(b \ell_{1} \ell_{3}\right),\left(t b \ell_{1}\right)$, and $\left(\ell_{1} \ell_{2} \ell_{3}\right)$. The asymmetries are calculated for $p p \rightarrow \tilde{t}_{1} \tilde{t}_{1}^{*}$ production at the LHC with the subsequent decays $\tilde{t}_{1} \rightarrow t \tilde{\chi}_{2}^{0}$ and $\tilde{\chi}_{2}^{0} \rightarrow \ell \tilde{\ell}_{R}$. The functional dependence of the asymmetries on the stop boost is purely kinematic. In the stop rest frame, $\beta_{\tilde{t}}=0$, they coincide with the corresponding epsilon product asymmetries, and then decrease with increasing $\beta_{\tilde{t}}$. Note that the size of the asymmetries strongly depends on the choice of momenta, and largest values are obtained for the optimal triple product $\left(t \ell_{1} \ell_{3}\right)$ as given in Eq. (8) . However this asymmetry also decreases most strongly with $\beta_{\tilde{t}}$, see Figure 7 (left). 
A triple product asymmetry in the laboratory frame is then obtained by folding the boost dependent asymmetry $\mathcal{A}$ with the normalized stop boost distribution,

$$
\mathcal{A}^{\mathrm{lab}}=\frac{1}{\sigma} \int_{0}^{1} \frac{d \sigma}{d \beta_{\tilde{t}}} \mathcal{A}\left(\beta_{\tilde{t}}\right) d \beta_{\tilde{t}}
$$

with the production cross section $\sigma=\sigma\left(p p \rightarrow \tilde{t}_{m} \tilde{t}_{m}^{*}\right)$. This leads to a reduction to roughly $30-40 \%$ of the asymmetry $\mathcal{A}^{\text {lab }}$ compared to $\mathcal{A}\left(\beta_{\tilde{t}}=0\right)$. This can be seen in Figure 7 (right), where we show both asymmetries as a function of the CP phase $\phi_{A_{t}}$. Thus the stop boost effectively reduces the asymmetries, but does not change their shape with respect to the phase dependence. In the following we will focus on the folded asymmetry $\mathcal{A}\left(t \ell_{1} \ell_{3}\right)$ in the laboratory frame but similar discussions hold for asymmetries based on the other triple products.

\section{Theoretical Statistical Significance}

So far we have only discussed the asymmetry of the triple product, but in order to determine the statistical significance of a given asymmetry it is also necessary to take into account the total signal rate. For example, a large asymmetry does not necessarily result in a large significance if the signal rate is low as this does increase the statistical uncertainty.

Assuming that the fluctuations of the signal rate are binomially distributed with the selection probability $p=1 / 2(\mathcal{A}+1)$, the significance of an asymmetry is

$$
\mathcal{S}=\frac{|\mathcal{A}|}{\sqrt{1-\mathcal{A}^{2}}} \sqrt{\sigma \mathcal{L}}
$$

with the integrated LHC luminosity $\mathcal{L}$. The cross section is

$$
\begin{aligned}
\sigma=F_{N} & \times \sigma\left(p p \rightarrow \tilde{t}_{m} \tilde{t}_{m}^{*}\right) \times \operatorname{Br}\left(\tilde{t}_{m} \rightarrow t \tilde{\chi}_{i}^{0}\right) \times \operatorname{Br}(t \rightarrow b W) \times \operatorname{Br}\left(W \rightarrow \nu_{e} e\right) \\
& \times \operatorname{Br}\left(\tilde{\chi}_{i}^{0} \rightarrow e^{+} \tilde{e}_{n}^{-}\right) \times \operatorname{Br}\left(\tilde{e}_{n}^{-} \rightarrow \tilde{\chi}_{1}^{0} e^{-}\right)
\end{aligned}
$$

for $m=1,2, i=2,3,4$, and $n=L, R$. The combinatorial factor $F_{N}$ takes into account the possible $W$ and neutralino $\tilde{\chi}_{i}^{0}$ decays into leptons with different flavors. We assume that the branching ratios do not depend on the flavor, i.e., $\operatorname{Br}(W \rightarrow$ $\left.\nu_{e} e\right)=\operatorname{Br}\left(W \rightarrow \nu_{\mu} \mu\right)$, and $\operatorname{Br}\left(\tilde{\chi}_{i}^{0} \rightarrow e^{+} \tilde{e}_{n}^{-}\right)=\operatorname{Br}\left(\tilde{\chi}_{i}^{0} \rightarrow \mu^{+} \tilde{\mu}_{n}^{-}\right)$. The factor is thus $F_{N}=8$, if we sum the lepton flavors $e, \mu$, and also the slepton charges, $\tilde{\ell}_{n}^{-}$and $\tilde{\ell}_{n}^{+}$.

The statistical significance $\mathcal{S}$ is equal to the number of standard deviations that the asymmetry can be determined to be non-zero. For example a value of $\mathcal{S}=1$ implies a measurement at the $68 \%$ confidence level. The minimal required luminosity is then

$$
\mathcal{L}=\frac{1}{\sigma}\left(\frac{1}{\mathcal{A}^{2}}-1\right)
$$

Our definition of the statistical significance $\mathcal{S}$ and the required luminosity $\mathcal{L}$ is purely based on the theoretical signal rate and its asymmetry. Detector efficiency 
effects and contributions from CP-even backgrounds are neglected, which would reduce the effective asymmetry. The definitions have thus to be regarded as absolute upper bounds on the confidence levels and as absolute lower bounds on the minimal required luminosities, respectively. We will use these terms in the following to exclude mSUGRA parameter regions which cannot be probed at the LHC. In order to give realistic values of the statistical significances and required luminosities to observe a CP signal, a detailed experimental study is necessary.

Also higher order corrections will be important and have to be included in a comprehensive analysis. Leading-order (LO) QCD calculations strongly depend on the a priori unknown renormalization and factorization scales. As a result, theoretical predictions of LO QCD cross sections and branching ratios are easily uncertain within a factor of 2 , see for example Ref. [53]. However, we expect that asymmetries and distributions will have much smaller scale dependencies. Since the higher order corrections enter both in the numerator and the denominator, they might cancel partially, and may lead to a significantly reduced scale dependence of the asymmetries. For instance, the normalized stop boost distribution shown in Fig. 6 (right) only changes slightly if switching on initial and final state radiations in PYTHIA [56]. Soft gluon radiation could in principle change the spin structure of the stop decay process, see Figure 1. However, only the top-neutralino spin-spin correlations are CP-sensitive, and thus they could only be altered by gluon radiation of the top alone.

Finally, our asymmetries probe CP-violating phases of the stop-top-neutralino couplings, which only belong to the electroweak sector. However, we expect that also the effect of electroweak corrections to our observables and asymmetries is rather small. Although electroweak corrections to neutralino masses, for example, can be $10 \%$ at one-loop level [57, and neutralino branching ratios for two-body decays may receive electroweak CP-even one-loop corrections of up to $16 \%$ in some cases [58, we expect that asymmetries are less sensitive to these electroweak corrections, since they again enter both in the numerator and denominator.

\subsection{Phase Dependence}

We analyze the $\phi_{A_{t}}$ and $\phi_{\mu}$ dependence of the triple product asymmetry $\mathcal{A}^{\text {lab }}$ in the lab frame, as defined in Eq. (21). For stop $p p \rightarrow \tilde{t}_{1} \tilde{t}_{1}^{*}$ production and subsequent decays $\tilde{t}_{1} \rightarrow t \tilde{\chi}_{2}^{0}$ and $\tilde{\chi}_{2}^{0} \rightarrow \ell \tilde{\ell}_{R}$, we show in Figure 8 (left) contour lines of $\mathcal{A}\left(t \ell_{1} \ell_{3}\right)$ in the $\phi_{A_{t}}-\phi_{\mu}$ plane for our reference scenario (Table 2). The maximal asymmetry in the lab frame is about $15 \%$, whereas the dependence on $\phi_{M_{1}}$ is weak, due to the negligible bino admixture of $\tilde{\chi}_{2}^{0}$ in mSUGRA scenarios. Note that the phase dependence of the asymmetry Figure 8 (left) closely resembles that of the effective coupling factor $\eta$, Eq. (F.79), shown in Figure 2 (right) for the same scenario. Thus the asymmetry directly probes the CP-violating structure of the stop-top-neutralino couplings. The kinematical part of the asymmetry is largely phase independent.

To determine the potential observability of the asymmetry, we calculate the minimal required luminosity $\mathcal{L}$, Eq. (24), to observe a $1 \sigma$ deviation of the asymmetry above the statistical fluctuations. Contour lines of $\mathcal{L}$ in the $\phi_{A_{t}}-\phi_{\mu}$ plane are shown 

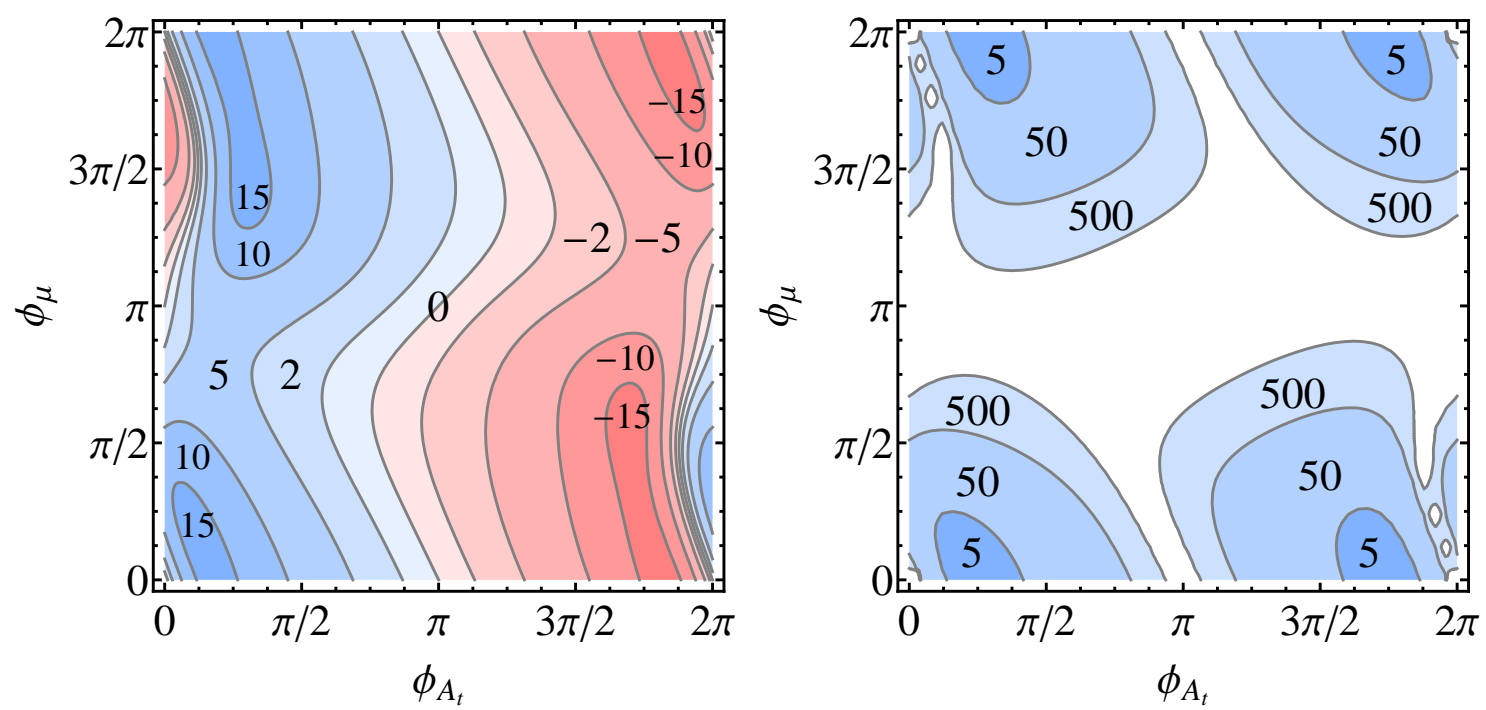

Figure 8: Triple product asymmetry $\mathcal{A}^{\text {lab }}\left(t \ell_{1} \ell_{3}\right)$, see Eq. (21), in percent (left), and minimal bound on the luminosity $\mathcal{L}$, Eq. (24), in $\mathrm{fb}^{-1}$ to detect such an asymmetry at $1 \sigma$ above fluctuations (right), as functions of the CP phases $\phi_{A_{t}}, \phi_{\mu}$, with $\phi_{M_{1}}=0$, for stop production at the LHC with $\sqrt{s_{p p}}=14 \mathrm{TeV}$ and decay $\tilde{t}_{1} \rightarrow t \tilde{\chi}_{2}^{0}, \tilde{\chi}_{2}^{0} \rightarrow \ell_{1} \tilde{\ell}_{R}$ see Figure 1. The mSUGRA parameters are given in Table 2 ,

in Figure 8 (right). A large part of the parameter space can be probed with $\mathcal{L}=$ $500 \mathrm{fb}^{-1}$. Even small phases close to the CP-conserving points $\phi_{A_{t}}, \phi_{\mu}=0, \pi$ can be probed with $\mathcal{L}=50 \mathrm{fb}^{-1}$. The significance also depends on the stop and neutralino branching ratios, see Eq. (23), which depend differently on the phases $\phi_{A_{t}}, \phi_{\mu}$. Thus the contour lines of the luminosity do not directly follow those of the asymmetry.

\section{4 mSUGRA Parameter Space}

So far we have analyzed the asymmetries within our mSUGRA scenario (Table 2). We now extend our study to the entire mSUGRA parameter space, and study the dependence of the asymmetries and the event rates on the parameters $m_{0}, m_{1 / 2}$, $\tan \beta$, and $A_{0}$. In such an mSUGRA framework, the production of light stops will dominate, $p p \rightarrow \tilde{t}_{1} \tilde{t}_{1}^{*}$, followed by the subsequent decays $\tilde{t}_{1} \rightarrow t \tilde{\chi}_{2}^{0}$, and $\tilde{\chi}_{2}^{0} \rightarrow \ell \tilde{\ell}_{R}$.

\section{Dependence on $m_{0}$ and $m_{1 / 2}$}

The asymmetries in the stop rest frame and in the LHC lab frame are rather independent of the masses of the stop and its decay products. Thus we find an almost constant asymmetry $\mathcal{A}^{\mathrm{lab}}\left(t \ell_{1} \ell_{3}\right) \approx 15 \%$ within the kinematical allowed region of the $m_{0}-m_{1 / 2}$ parameter plane. However the stop production rate and the branching ratios of the neutralinos sensitively depend on $m_{0}$ and $m_{1 / 2}$.

In Figure 9 (left), we show contour lines of the leading order (LO) cross section $\sigma\left(p p \rightarrow \tilde{t}_{1} \tilde{t}_{1}^{*}\right) \times \operatorname{Br}\left(\tilde{t}_{1} \rightarrow t \tilde{\chi}_{2}^{0}\right)$ in the $m_{0}-m_{1 / 2}$ plane. While the stop decay branching ratio $\operatorname{Br}\left(\tilde{t}_{1} \rightarrow t \tilde{\chi}_{2}^{0}\right) \approx 0.1$ is almost constant, the stop production cross section 

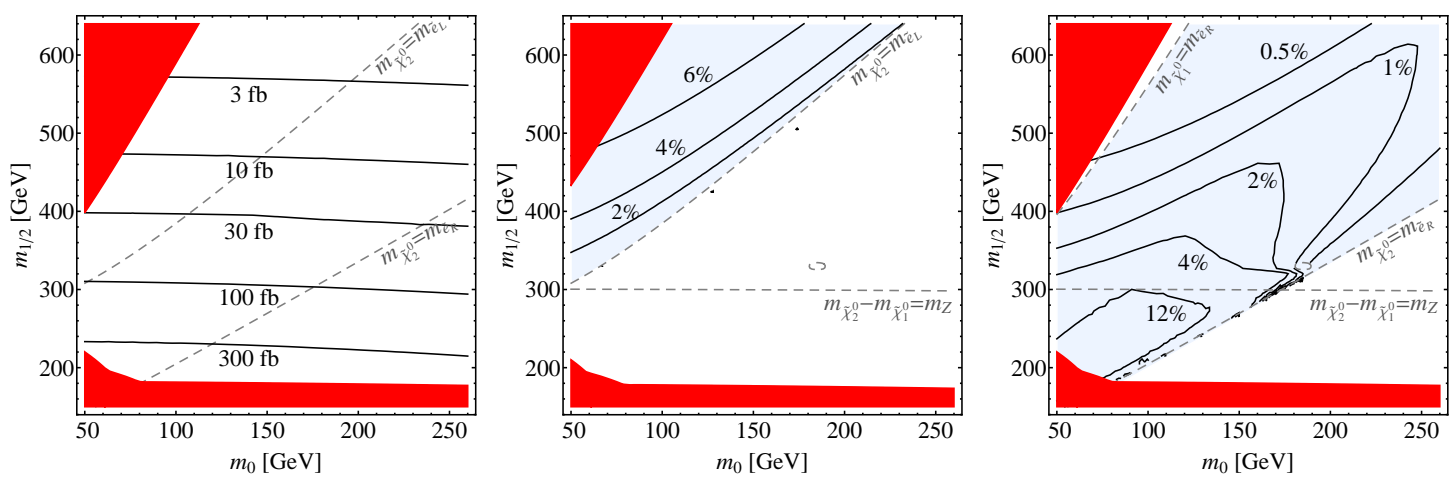

Figure 9: Cross section $\sigma\left(p p \rightarrow \tilde{t}_{1} \tilde{t}_{1}^{*}\right) \times \operatorname{Br}\left(\tilde{t}_{1} \rightarrow t \tilde{\chi}_{2}^{0}\right)$ (left), neutralino branching ratio into left slepton $\operatorname{Br}\left(\tilde{\chi}_{2}^{0} \rightarrow e^{+} \tilde{e}_{L}^{-}\right)$(middle), and $\tilde{\chi}_{2}^{0}$ branching ratio into right slepton $\operatorname{Br}\left(\tilde{\chi}_{2}^{0} \rightarrow e^{+} \tilde{e}_{R}^{-}\right)$(right), as a function of the mSUGRA parameters $m_{0}$ and $m_{1 / 2}$. The other mSUGRA parameters and the CP phases are given in Table 2. The dark red area is excluded by direct SUSY searches.

strongly decreases with increasing stop mass, see Figure 6 (left). Therefore the cross section also rapidly drops with increasing $m_{1 / 2}$, which clearly can be seen in Figure 9 (left). The dashed lines $m_{\chi_{2}^{0}}=m_{\tilde{e}_{R, L}}$ indicate the neutralino decay thresholds into sleptons. We show their branching ratios $\operatorname{Br}\left(\tilde{\chi}_{2}^{0} \rightarrow e^{+} \tilde{e}_{L}^{-}\right)$and $\operatorname{Br}\left(\tilde{\chi}_{2}^{0} \rightarrow e^{+} \tilde{e}_{R}^{-}\right)$ in Figure 9 (middle) and (right), respectively. The branching ratio into right selectrons reaches up to $12 \%$ for small values of $m_{0}$ and $m_{1 / 2}$. The decay fraction is considerably reduced for $m_{1 / 2}>300 \mathrm{GeV}$, where decays into the $Z$ and the lightest neutral Higgs boson open, see the dashed line $m_{\chi_{2}^{0}}-m_{\chi_{1}^{0}}=m_{Z}$ in Figure 9. The significance for observing an asymmetry for the decay $\tilde{\chi}_{2}^{0} \rightarrow \ell \tilde{\ell}_{L}$ is suppressed to that for the decay $\tilde{\chi}_{2}^{0} \rightarrow \ell \tilde{\ell}_{R}$ for most of the $m_{0}-m_{1 / 2}$ parameter space. This is because the branching ratio $\operatorname{Br}\left(\tilde{\chi}_{2}^{0} \rightarrow \ell \tilde{\ell}_{L}\right)$ is larger for higher values of $m_{1 / 2}$, where however the stop production cross section is suppressed. We thus find that both $m_{0}$ and $m_{1 / 2}$ should be as small as possible to obtain the highest process rates.

These findings are confirmed when we calculate the minimal luminosity $\mathcal{L}$, Eq. (24), which is required to observe the asymmetry at $1 \sigma$ for stop production $p p \rightarrow \tilde{t}_{1} \tilde{t}_{1}^{*}$ at the LHC, with the subsequent decays $\tilde{t}_{1} \rightarrow t \tilde{\chi}_{2}^{0}$ and $\tilde{\chi}_{2}^{0} \rightarrow \ell \tilde{\ell}_{L(R)}$, see Figure 10 (left) and (right), respectively. The mSUGRA parameters and the $\mathrm{CP}$ phases are given in Table 2. For right sleptons, the required integrated luminosity can be as low as $2 \mathrm{fb}^{-1}$ for $m_{0} \approx 50-100 \mathrm{GeV}$ and $m_{1 / 2} \approx 200 \mathrm{GeV}$. Asymmetries involving left sleptons require at least $\mathcal{L} \approx 150 \mathrm{fb}^{-1}$ for $m_{0} \approx 50 \mathrm{GeV}$ and $m_{1 / 2} \approx 340-420 \mathrm{GeV}$. In the overlap between the allowed kinematical regions for decays into left and right sleptons, the rates can be comparable, though. As the two asymmetries have the same magnitude but opposite signs, $\mathcal{A}^{\tilde{\ell}_{L}}\left(t \ell_{1} \ell_{3}\right)=-\mathcal{A}^{\tilde{\ell}_{R}}\left(t \ell_{1} \ell_{3}\right)$, this would lead to a reduction of the total asymmetry in this intermediate region. In Figure 10, we also indicate the $m_{0}-m_{1 / 2}$ parameter region where three-body decays $\tilde{\chi}_{2}^{0} \rightarrow \ell^{+} \ell^{-} \tilde{\chi}_{1}^{0}$ have been used to study CP asymmetries [43]. 

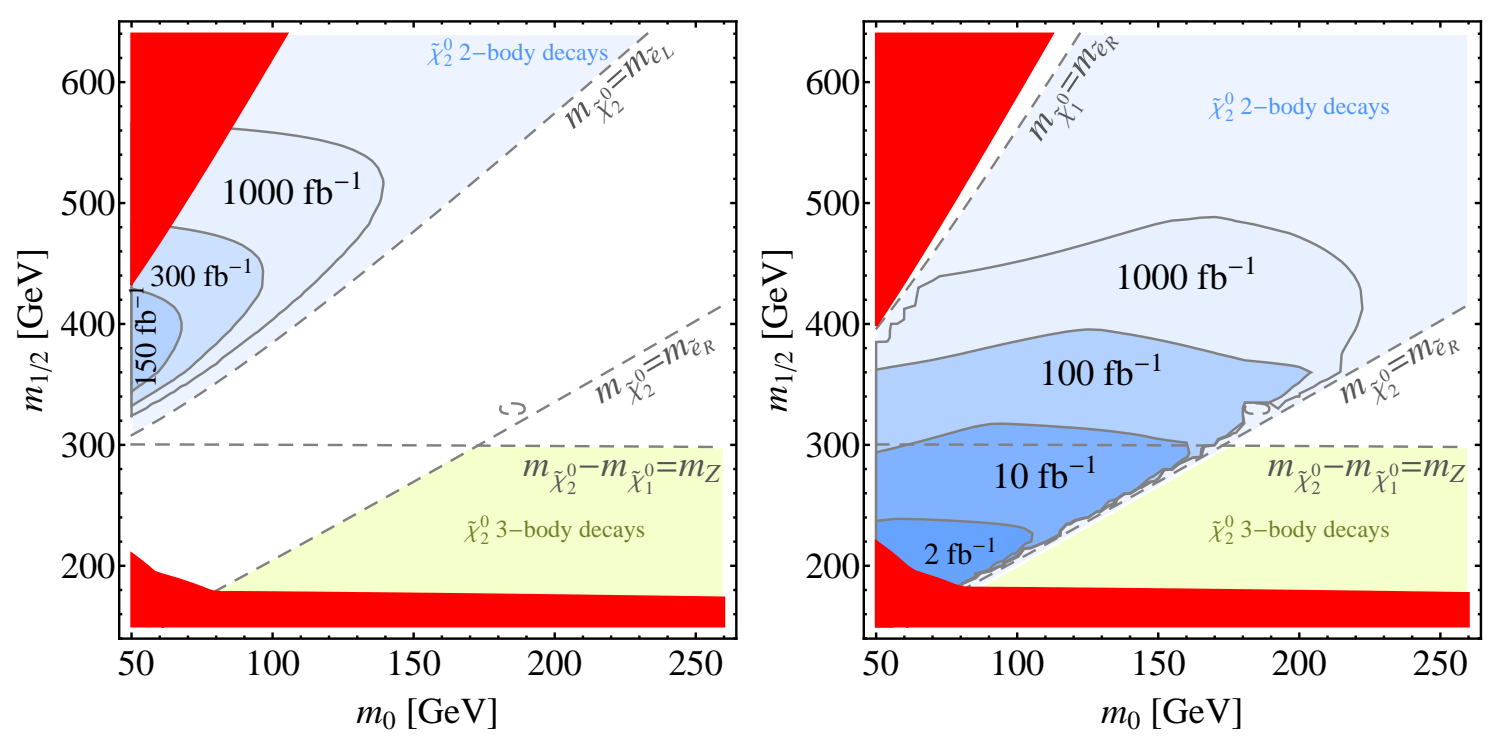

Figure 10: Contour lines in the $m_{0}-m_{1 / 2}$ plane of the minimal required luminosity $\mathcal{L}$, Eq. (24), to observe the asymmetry $\mathcal{A}^{\text {lab }}\left(t \ell_{1} \ell_{3}\right)$, Eq. (21), at $1 \sigma$ above fluctuations for stop production at the LHC with $\sqrt{s_{p p}}=14 \mathrm{TeV}$ and decay $\tilde{t}_{1} \rightarrow t \tilde{\chi}_{2}^{0}$, via a left slepton $\tilde{\chi}_{2}^{0} \rightarrow \ell_{1} \tilde{\ell}_{L}$ (left) and right slepton $\tilde{\chi}_{2}^{0} \rightarrow \ell_{1} \tilde{\ell}_{R}$ (right), see Figure 1. The other mSUGRA parameters and the CP phases are given in Table 2 . The dark red area is excluded by direct SUSY searches.

\section{Dependence on $A_{0}$ and $\tan \beta$}

In Figure 11, we show contour lines in the $A_{0}-\tan \beta$ plane of the asymmetry $\mathcal{A}\left(t \ell_{1} \ell_{3}\right)$ for stop production, $p p \rightarrow \tilde{t}_{1} \tilde{t}_{1}^{*}$, (and decay $\tilde{t}_{1} \rightarrow t \tilde{\chi}_{2}^{0}, \tilde{\chi}_{2}^{0} \rightarrow \ell \tilde{\ell}_{R}$, see Figure 1) and the corresponding minimal luminosity to observe it at $1 \sigma$ above fluctuations. All other parameters are given as in the reference scenario, Table 2 . The asymmetry is essentially independent of $A_{0}$ and $\tan \beta$ over the region of interest, slightly peaking at $16.5 \%$ at $A_{0} \approx 500 \mathrm{GeV}$ and $\tan \beta \approx 10-15$. For small values of $\tan \beta \lesssim 2$, a reduced stop mixing leads to smaller asymmetries. On the other hand, an increasing value of $A_{0}$ also enhances the stau neutralino branching ratios, $\tilde{\chi}_{2}^{0} \rightarrow \tau \tilde{\tau}$. As a result of this, the dependence of the required luminosity on $A_{0}$ and $\tan \beta$ is dominated by the rate, and the lowest luminosities are achieved for small values of $A_{0}$ and $\tan \beta$, see Figure 11 (right).

\subsection{Angular and Volume Distributions of Triple Products}

A measurement of an asymmetry requires the reconstruction of the spatial momenta of the three particles which form the triple product. It has to be determined whether the three momenta form a left- or right-handed trihedron. This can certainly only be answered by a detailed experimental simulation for the LHC. However to gain some insight, we briefly discuss the distributions of the stop decay width with respect to the volume and opening angles of the trihedron spanned by the momenta. 

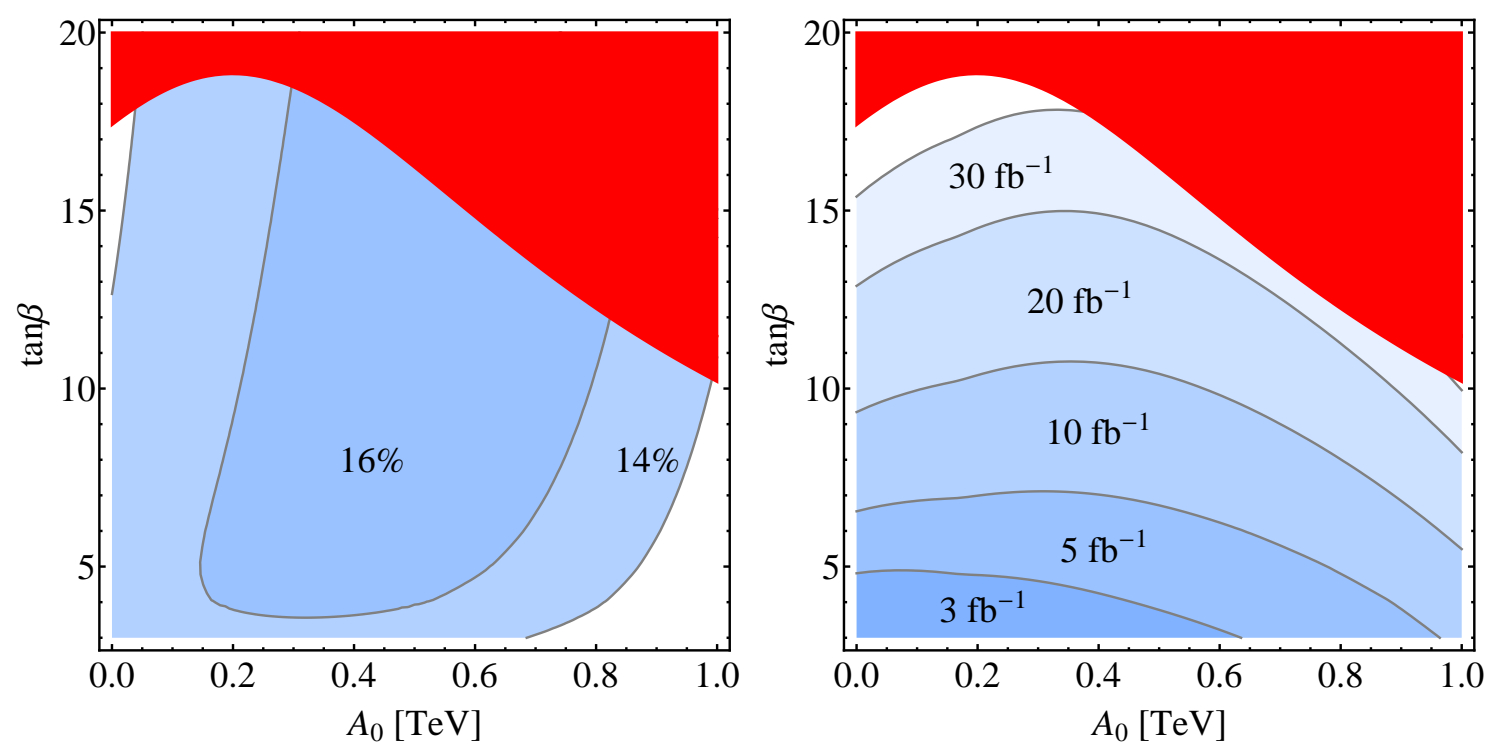

Figure 11: Contour lines in the $A_{0}-\tan \beta$ plane of the asymmetry $\mathcal{A}^{\text {lab }}\left(t \ell_{1} \ell_{3}\right)$, Eq. (21), in the laboratory frame (left), and the minimal required luminosity $\mathcal{L}$, Eq. (24), to observe it at $1 \sigma$ above fluctuations (right), for stop production at the LHC with $\sqrt{s_{p p}}=14 \mathrm{TeV}$ and decay $\tilde{t}_{1} \rightarrow t \tilde{\chi}_{2}^{0}, \tilde{\chi}_{2}^{0} \rightarrow \ell_{1} \tilde{\ell}_{R}$, see Figure 1. The other mSUGRA parameters and CP phases are given in Table 2. The dark red area is excluded by direct SUSY searches.
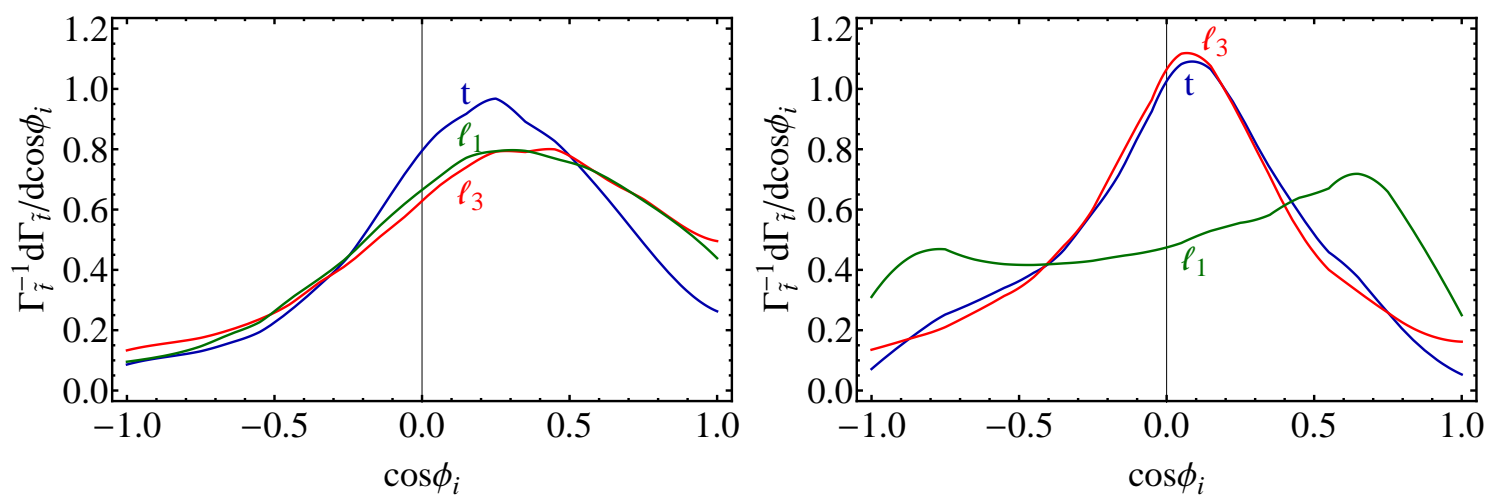

Figure 12: Normalized distributions of the stop decay width $\Gamma$ with respect to the cosine of the three opening angles $\phi_{i}\left(i=t, \ell_{1}, \ell_{3}\right)$ of the parallelepiped $\left(\mathbf{p}_{t}, \mathbf{p}_{\ell_{1}}, \mathbf{p}_{\ell_{3}}\right)$, e.g. $\phi_{t}=\Varangle\left(\mathbf{p}_{t}, \mathbf{p}_{\ell_{1}} \times \mathbf{p}_{\ell_{3}}\right)$. Results are shown for stop boost factors $\beta_{\tilde{t}_{1}}=0$ (left), and $\beta_{\tilde{t}_{1}}=0.8$ (right). The mSUGRA parameters and CP phases are given in Table 2.

In Figure 12, we show the distribution of the stop decay width, $d \Gamma / d \cos \phi_{i}$, with respect to one of the three opening angles of the trihedron formed by the momenta $\mathbf{p}_{t}, \mathbf{p}_{\ell_{1}}, \mathbf{p}_{\ell_{3}}$, where $\phi_{i}\left(i=t, \ell_{1}, \ell_{3}\right)$ is the opening angle between one particle momentum and the plane formed by the other two, e.g., $\phi_{t}=\Varangle\left(\mathbf{p}_{t}, \mathbf{p}_{\ell_{1}} \times \mathbf{p}_{\ell_{3}}\right)$. In Figure 12 (left), the distribution is shown in the stop rest frame, and in Figure 12 (right) for a boost factor $\beta_{\tilde{t}_{1}}=0.8$. The parallelepiped collapses to a plane for 


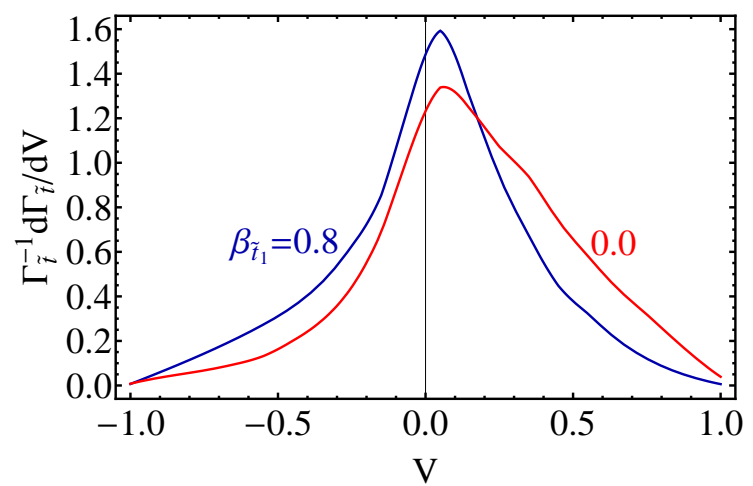

Figure 13: Normalized distributions of the stop decay width $\Gamma$ with respect to the normalized volume $V=\mathbf{p}_{t} \cdot\left(\mathbf{p}_{\ell_{1}} \times \mathbf{p}_{\ell_{3}}\right) /\left(\left|\mathbf{p}_{t}\right|\left|\mathbf{p}_{\ell_{1}}\right|\left|\mathbf{p}_{\ell_{3}}\right|\right)$ of the three particle momenta which form the parallelepiped. Results are shown for stop boost factors $\beta_{\tilde{t}_{1}}=0$ and $\beta_{\tilde{t}_{1}}=0.8$. The mSUGRA parameters and CP phases are given in Table 2 .

$\cos \phi_{i}=0$. For angles close to this configuration it will be difficult to reconstruct the handedness of the trihedron. For larger stop boost factors, the distributions become even more symmetric and the peaks move closer to $\cos \phi_{i}=0$. Only for $\phi_{\ell_{1}}=\Varangle\left(\mathbf{p}_{\ell_{1}}, \mathbf{p}_{\ell_{3}} \times \mathbf{p}_{t}\right)$, Figure 12 (right), double peaks are formed at $\phi_{\ell_{1}} \approx \pm 45^{\circ}$, which should simplify the determination of the handedness in a boosted frame.

In Figure 13, we show the normalized distribution $d \Gamma / d V$ with respect to the normalized volume $V=\hat{\mathbf{p}}_{t} \cdot\left(\hat{\mathbf{p}}_{\ell_{1}} \times \hat{\mathbf{p}}_{\ell_{3}}\right)$ of the unit vectors $\hat{\mathbf{p}}=\mathbf{p} /|\mathbf{p}|$ for a stop boost factor $\beta_{\tilde{t}_{1}}=0$ and $\beta_{\tilde{t}_{1}}=0.8$. This succinctly demonstrates that the event configurations mainly have the form of a collapsed parallelepiped, in particular for large stop boosts.

\section{$5 \quad$ Summary and Conclusions}

We have analyzed observables of CP violation in the two-body decays of a light stop

$$
\tilde{t}_{1} \rightarrow t+\tilde{\chi}_{2}^{0}
$$

The CP-sensitive parts appear only in the top neutralino spin-spin correlations, which can be probed by the subsequent decays

$$
\begin{aligned}
t & \rightarrow b+W, \quad W \rightarrow \nu_{\ell}+\ell_{3} \\
\tilde{\chi}_{2}^{0} & \rightarrow \ell_{1}+\tilde{\ell}_{R}, \quad \tilde{\ell}_{R} \rightarrow \tilde{\chi}_{1}^{0}+\ell_{2}, \quad \ell=e, \mu .
\end{aligned}
$$

This specific decay chain of the light stop was found to give the largest signal of CP violation over most of the mSUGRA parameter space.

Due to angular momentum conservation, the decay distributions of the final state momenta are correlated to each other. Asymmetries of triple products of three spatial momenta, as well as epsilon products of four space-time momenta are 
ideal CP observables. They are sensitive to the CP phases of the trilinear coupling parameter $A_{t}$, the higgsino mass parameter $\mu$, and the gaugino mass parameter $M_{1}$.

We have analyzed the asymmetries and event rates in an mSUGRA framework, with CP-violating phases explicitly added at the weak scale. For a strong stop mixing, the asymmetries are rather constant in the mSUGRA space, and reach up to $40 \%$ in the rest frame of the stop for $\phi_{A_{t}}=1 / 5 \pi$ and $\phi_{\mu}=0$. The influence of $\phi_{M_{1}}$ is negligible for a wino-like neutralino.

The asymmetries of triple products are not Lorentz invariant and therefore framedependent. Since the stops are produced highly boosted at the LHC, the asymmetries are reduced by a factor of about three when evaluated in the laboratory frame, compared to the stop rest frame. Luminosities of at least $10 \mathrm{fb}^{-1}$ for small values of $m_{1 / 2} \lesssim 300 \mathrm{GeV}, m_{0} \lesssim 150 \mathrm{GeV}$ and $\tan \beta \approx 5$ are required to observe a $\mathrm{CP}$ signal of $1 \sigma$ above statistical fluctuations at the LHC.

There are already stringent constraints on SUSY CP phases from electric dipole moments, but large phases and CP asymmetries at the LHC are possible when taking into account cancellations between EDM contributions from different phases. Since the asymmetries of T-odd products depend differently than EDMs on the SUSY parameters, in particular on the $\mathrm{CP}$ phases, asymmetries would be an ideal tool for independent measurements at the LHC and ILC.

Clearly, the measurability of the asymmetries and thus of the $\mathrm{CP}$ phases can only be addressed properly in a detailed experimental analysis, which should take into account background processes, detector simulations and event reconstruction efficiencies. We want to underline the need for such a thorough analysis, to explore the potential of the LHC to probe SUSY CP violation. 


\section{Acknowledgments}

We thank J. A. Aguilar-Saavedra, F. del Águila, N. F. Castro, S. Heinemeyer, T. Kernreiter, U. Nierste, A. Pilaftsis, and J. Reuter for very helpful comments and discussions. OK would like to thank the School of Physics and Astronomy of the University of Manchester for kind hospitality, and J. S. Kim, aka Zong, for assistance in using MadGraph [54]. FFD would like to thank the Department of Physics and Astronomy of the University of Granada for kind hospitality. This work is supported by MICINN project FPA.2006-05294. 


\section{Appendix}

\section{A Stop Mixing}

The masses and couplings of the stops follow from their mass matrix [4]

$$
\mathcal{L}_{M}^{\tilde{t}}=-\left(\tilde{t}_{L}^{*}, \tilde{t}_{R}^{*}\right)\left(\begin{array}{cc}
m_{\tilde{t}_{L}}^{2} & e^{-i \phi_{\tilde{t}} m_{t}\left|\Lambda_{\tilde{t}}\right|} \\
e^{i \phi_{\tilde{t}} m_{t}\left|\Lambda_{\tilde{t}}\right|} & m_{\tilde{t}_{R}}^{2}
\end{array}\right)\left(\begin{array}{c}
\tilde{t}_{L} \\
\tilde{t}_{R}
\end{array}\right),
$$

with

$$
\begin{aligned}
& m_{\tilde{t}_{L}}^{2}=M_{\tilde{Q}}^{2}+\left(\frac{1}{2}-\frac{2}{3} \sin ^{2} \theta_{w}\right) m_{Z}^{2} \cos 2 \beta+m_{t}^{2} \\
& m_{\tilde{t}_{R}}^{2}=M_{\tilde{U}}^{2}+\frac{2}{3} m_{Z}^{2} \sin ^{2} \theta_{w} \cos 2 \beta+m_{t}^{2}
\end{aligned}
$$

with the soft SUSY-breaking parameters $M_{\tilde{Q}}, M_{\tilde{U}}$, the ratio $\tan \beta=\frac{v_{2}}{v_{1}}$ of the vacuum expectation values of the two neutral Higgs fields, the weak mixing angle $\theta_{w}$, the mass $m_{Z}$ of the $Z$ boson, and the mass $m_{t}$ of the top quark. The CP phase of the stop sector is

$$
\begin{aligned}
\phi_{\tilde{t}} & =\arg \left[\Lambda_{\tilde{t}}\right], \\
\Lambda_{\tilde{t}} & =A_{t}-\mu^{*} \cot \beta,
\end{aligned}
$$

with the complex trilinear scalar coupling parameter $A_{t}$, and the higgsino mass parameter $\mu$. The stop mass eigenstates

$$
\left(\begin{array}{c}
\tilde{t}_{1} \\
\tilde{t}_{2}
\end{array}\right)=\mathcal{R}^{\tilde{t}}\left(\begin{array}{c}
\tilde{t}_{L} \\
\tilde{t}_{R}
\end{array}\right)
$$

are given by the diagonalization matrix [4]

$$
\mathcal{R}^{\tilde{t}}=\left(\begin{array}{cc}
e^{i \phi_{\tilde{t}}} \cos \theta_{\tilde{t}} & \sin \theta_{\tilde{t}} \\
-\sin \theta_{\tilde{t}} & e^{-i \phi_{\tilde{t}}} \cos \theta_{\tilde{t}}
\end{array}\right)
$$

with the stop mixing angle $\theta_{\tilde{t}}$

$$
\cos \theta_{\tilde{t}}=\frac{-m_{t}\left|\Lambda_{\tilde{t}}\right|}{\sqrt{m_{t}^{2}\left|\Lambda_{\tilde{t}}\right|^{2}+\left(m_{\tilde{t}_{1}}^{2}-m_{\tilde{t}_{L}}^{2}\right)^{2}}}, \quad \sin \theta_{\tilde{t}}=\frac{m_{\tilde{t}_{L}}^{2}-m_{\tilde{t}_{1}}^{2}}{\sqrt{m_{t}^{2}\left|\Lambda_{\tilde{t}}\right|^{2}+\left(m_{\tilde{t}_{1}}^{2}-m_{\tilde{t}_{L}}^{2}\right)^{2}}}
$$

The mass eigenvalues are

$$
m_{\tilde{t}_{1,2}}^{2}=\frac{1}{2}\left[\left(m_{\tilde{t}_{L}}^{2}+m_{\tilde{t}_{R}}^{2}\right) \mp \sqrt{\left(m_{\tilde{t}_{L}}^{2}-m_{\tilde{t}_{R}}^{2}\right)^{2}+4 m_{t}^{2}\left|\Lambda_{\tilde{t}}\right|^{2}}\right] .
$$

Note that for $\left|A_{t}\right| \gg|\mu| \cot \beta$ we have $\phi_{\tilde{t}} \approx \phi_{A_{t}}$. 


\section{B Neutralino Mixing}

The complex symmetric mass matrix of the neutralinos in the photino, zino, higgsino basis $\left(\tilde{\gamma}, \tilde{Z}, \tilde{H}_{a}^{0}, \tilde{H}_{b}^{0}\right)$, is given by [59]

$$
\mathcal{M}_{\chi^{0}}=\left(\begin{array}{cccc}
M_{2} s_{w}^{2}+M_{1} c_{w}^{2} & \left(M_{2}-M_{1}\right) s_{w} c_{w} & 0 & 0 \\
\left(M_{2}-M_{1}\right) s_{w} c_{w} & M_{2} c_{w}^{2}+M_{1} s_{w}^{2} & m_{Z} & 0 \\
0 & m_{Z} & \mu s_{2 \beta} & -\mu c_{2 \beta} \\
0 & 0 & -\mu c_{2 \beta} & -\mu s_{2 \beta}
\end{array}\right)
$$

with the short hand notation for the angles $s_{w}=\sin \theta_{w}, c_{w}=\cos \theta_{w}$, and $s_{2 \beta}=$ $\sin (2 \beta), c_{2 \beta}=\cos (2 \beta)$, and the $S U(2)$ gaugino mass parameter $M_{2}$. The phases of the complex parameters $M_{1}=\left|M_{1}\right| e^{i \phi_{M_{1}}}$ and $\mu=|\mu| e^{i \phi_{\mu}}$ can lead to CP-violating effects in the neutralino system. We diagonalize the neutralino mass matrix with a complex, unitary $4 \times 4$ matrix $N$ [1],

$$
N^{*} \cdot \mathcal{M}_{\chi^{0}} \cdot N^{\dagger}=\operatorname{diag}\left(m_{\chi_{1}^{0}}, \ldots, m_{\chi_{4}^{0}}\right)
$$

with the real neutralino masses $0<m_{\chi_{1}^{0}}<m_{\chi_{2}^{0}}<m_{\chi_{3}^{0}}<m_{\chi_{4}^{0}}$. This can be achieved by diagonalizing the hermitian matrix $\mathcal{M}_{\chi^{0}}^{\dagger} \cdot \mathcal{M}_{\chi^{0}}$ with a unitary matrix $U$

$$
U \cdot\left(\mathcal{M}_{\chi^{0}}^{\dagger} \cdot \mathcal{M}_{\chi^{0}}\right) \cdot U^{\dagger}=\operatorname{diag}\left(m_{\chi_{1}^{0}}^{2}, \ldots, m_{\chi_{4}^{0}}^{2}\right) .
$$

The neutralino mixing matrix $N$ can then be expressed as

$$
N=\operatorname{diag}\left(e^{i \phi_{1} / 2}, \ldots, e^{i \phi_{4} / 2}\right) \cdot U
$$

with the phases $\phi_{i}$ of the elements of the diagonal matrix $U^{*} \cdot \mathcal{M}_{\chi^{0}} \cdot U^{\dagger}$,

$$
\phi_{i}=\arg \left(U^{*} \cdot \mathcal{M}_{\chi^{0}} \cdot U^{\dagger}\right)_{i i}, \quad i=1, \ldots, 4 .
$$

\section{Lagrangians and Couplings}

The interaction Lagrangian for stop decay $\tilde{t}_{m} \rightarrow t \tilde{\chi}_{i}^{0}$ is given by [41]

$$
\mathscr{L}_{t \tilde{t} \tilde{\chi}^{0}}=g \bar{t}\left(a_{m i}^{\tilde{t}} P_{R}+b_{m i}^{\tilde{t}} P_{L}\right) \tilde{\chi}_{i}^{0} \tilde{t}_{m}+\text { h.c. }
$$

with $P_{L, R}=\left(1 \mp \gamma_{5}\right) / 2$, and the weak coupling constant $g=e / \sin \theta_{w}, e>0$. The couplings are

$$
a_{m i}^{\tilde{t}}=\sum_{n=1}^{2}\left(\mathcal{R}_{m n}^{\tilde{t}}\right)^{*} \mathcal{A}_{i n}^{t}, \quad b_{m i}^{\tilde{t}}=\sum_{n=1}^{2}\left(\mathcal{R}_{m n}^{\tilde{t}}\right)^{*} \mathcal{B}_{i n}^{t}
$$

with the stop diagonalization matrix $\mathcal{R}^{\tilde{t}}$, see Eq. (A.7), and

$$
\mathcal{A}_{i}^{t}=\left(\begin{array}{c}
f_{t i}^{L} \\
h_{t i}^{R}
\end{array}\right), \quad \mathcal{B}_{i}^{t}=\left(\begin{array}{c}
h_{t i}^{L} \\
f_{t i}^{R}
\end{array}\right),
$$


with, in the photino, zino, higgsino basis $\left(\tilde{\gamma}, \tilde{Z}, \tilde{H}_{a}^{0}, \tilde{H}_{b}^{0}\right)$,

$$
\begin{aligned}
f_{t i}^{L} & =-\sqrt{2}\left[\frac{1}{\cos \theta_{w}}\left(\frac{1}{2}-\frac{2}{3} \sin ^{2} \theta_{w}\right) N_{i 2}+\frac{2}{3} \sin \theta_{w} N_{i 1}\right], \\
f_{t i}^{R} & =-\frac{2 \sqrt{2}}{3} \sin \theta_{w}\left(\tan \theta_{w} N_{i 2}^{*}-N_{i 1}^{*}\right), \\
h_{t i}^{L} & =\left(h_{t i}^{R}\right)^{*}=Y_{t}\left(N_{i 3}^{*} \sin \beta-N_{i 4}^{*} \cos \beta\right), \\
Y_{t} & =\frac{m_{t}}{\sqrt{2} m_{W} \sin \beta},
\end{aligned}
$$

and $m_{W}$ the mass of the $W$ boson.

The interaction Lagrangian for neutralino decay $\tilde{\chi}_{i}^{0} \rightarrow \tilde{\ell}_{R, L}^{ \pm} \ell^{\mp}$, followed by $\tilde{\ell}_{R, L}^{ \pm} \rightarrow$ $\tilde{\chi}_{1}^{0} \ell^{ \pm}$with $\ell=e, \mu$ is given by [59]

$$
\mathscr{L}_{\ell \tilde{\ell} \tilde{\chi}^{0}}=g \bar{\ell} f_{\ell i}^{L} P_{R} \tilde{\chi}_{i}^{0} \tilde{\ell}_{L}+g \bar{\ell} f_{\ell i}^{R} P_{L} \tilde{\chi}_{i}^{0} \tilde{\ell}_{R}+\text { h.c. },
$$

with the couplings [59]

$$
\begin{aligned}
f_{\ell i}^{L} & =\sqrt{2}\left[\frac{1}{\cos \theta_{w}}\left(\frac{1}{2}-\sin ^{2} \theta_{w}\right) N_{i 2}+\sin \theta_{w} N_{i 1}\right], \\
f_{\ell i}^{R} & =\sqrt{2} \sin \theta_{w}\left(\tan \theta_{w} N_{i 2}^{*}-N_{i 1}^{*}\right) .
\end{aligned}
$$

The Lagrangian for $\ell=\tau$ with complex $A_{\tau}$ are given, for example, in Ref [27].

\section{Kinematics and Phase Space}

For the squark decay $\tilde{t}_{m} \rightarrow t \tilde{\chi}_{i}^{0}$, we choose a coordinate frame in the laboratory (lab) frame such that the momentum of the top squark $\tilde{t}_{m}$ points in the $z$-direction

$$
\begin{aligned}
p_{\tilde{t}}^{\mu} & =\left(E_{\tilde{t}}, 0,0,\left|\mathbf{p}_{\tilde{t}}\right|\right) \\
p_{t}^{\mu} & =\left(E_{t},\left|\mathbf{p}_{t}\right| \sin \theta_{t}, 0,\left|\mathbf{p}_{t}\right| \cos \theta_{t}\right)
\end{aligned}
$$

The decay angle $\theta_{t}=\Varangle\left(\mathbf{p}_{\tilde{t}}, \mathbf{p}_{t}\right)$ of the top quark is constrained by $\sin \theta_{t}^{\max }=\left|\mathbf{p}_{\tilde{t}}^{\prime}\right| /\left|\mathbf{p}_{\tilde{t}}\right|$ for $\left|\mathbf{p}_{\tilde{t}}\right|>\left|\mathbf{p}_{\tilde{t}}^{\prime}\right|=\lambda^{\frac{1}{2}}\left(m_{\tilde{t}}^{2}, m_{t}^{2}, m_{\chi_{i}^{0}}^{2}\right) / 2 m_{t}$, with the triangle function $\lambda(a, b, c)=a^{2}+$ $b^{2}+c^{2}-2(a b+a c+b c)$. In this case there are two solutions [36, 60.

$$
\left|\mathbf{p}_{t}^{ \pm}\right|=\frac{\left(m_{\tilde{t}}^{2}+m_{t}^{2}-m_{\chi_{i}^{0}}^{2}\right)\left|\mathbf{p}_{\tilde{t}}\right| \cos \theta_{t} \pm E_{\tilde{t}} \sqrt{\lambda\left(m_{\tilde{t}}^{2}, m_{t}^{2}, m_{\chi_{i}^{0}}^{2}\right)-4\left|\mathbf{p}_{\tilde{t}}\right|^{2} m_{t}^{2} \sin ^{2} \theta_{t}}}{2\left|\mathbf{p}_{\tilde{t}}\right|^{2} \sin ^{2} \theta_{t}+2 m_{\tilde{t}}^{2}} .
$$

For $\left|\mathbf{p}_{\tilde{t}}\right|<\left|\mathbf{p}_{\tilde{t}}^{\prime}\right|$ the angle $\theta_{t}$ is unbounded, and only the physical solution $\left|\mathbf{p}_{t}^{+}\right|$is left. 
The momenta of the subsequent decays of the neutralino $\tilde{\chi}_{i}^{0} \rightarrow \ell_{1} \tilde{\ell} ; \tilde{\ell} \rightarrow \tilde{\chi}_{1}^{0} \ell_{2}$ (3), and those of the top quark $t \rightarrow b W, W \rightarrow \ell_{3} \nu_{\ell}$ (4), can be parametrized by

$$
\begin{aligned}
p_{b}^{\mu} & =E_{b}\left(1, \sin \theta_{b} \cos \phi_{b}, \sin \theta_{b} \sin \phi_{b}, \cos \theta_{b}\right), \\
p_{\ell_{1}}^{\mu} & =E_{\ell_{1}}\left(1, \sin \theta_{1} \cos \phi_{1}, \sin \theta_{1} \sin \phi_{1}, \cos \theta_{1}\right), \\
p_{\ell_{2}}^{\mu} & =E_{\ell_{2}}\left(1, \sin \theta_{2} \cos \phi_{2}, \sin \theta_{2} \sin \phi_{2}, \cos \theta_{2}\right), \\
p_{\ell_{3}}^{\mu} & =E_{\ell_{3}}\left(1, \sin \theta_{3} \cos \phi_{3}, \sin \theta_{3} \sin \phi_{3}, \cos \theta_{3}\right),
\end{aligned}
$$

with the energies 36

$$
\begin{aligned}
E_{\ell_{1}} & =\frac{m_{\chi_{i}^{0}}^{2}-m_{\tilde{\ell}}^{2}}{2\left(E_{\chi_{i}^{0}}-\left|\mathbf{p}_{\chi_{i}^{0}}\right| \cos \theta_{D_{1}}\right)}, \quad E_{\ell_{2}}=\frac{m_{\tilde{\ell}}^{2}-m_{\chi_{1}^{0}}^{2}}{2\left(E_{\tilde{\ell}}-\left|\mathbf{p}_{\tilde{\ell}}\right| \cos \theta_{D_{2}}\right)}, \\
E_{b} & =\frac{m_{t}^{2}-m_{W}^{2}}{2\left(E_{t}-\left|\mathbf{p}_{t}\right| \cos \theta_{D_{b}}\right)}, \quad E_{\ell_{3}}=\frac{m_{W}^{2}}{2\left(E_{W}-\left|\mathbf{p}_{W}\right| \cos \theta_{D_{3}}\right)},
\end{aligned}
$$

and the decay angles, $\theta_{D_{1}}=\Varangle\left(\mathbf{p}_{\chi_{i}^{0}}, \mathbf{p}_{\ell_{1}}\right), \theta_{D_{2}}=\Varangle\left(\mathbf{p}_{\tilde{\ell}}, \mathbf{p}_{\ell_{2}}\right), \theta_{D_{3}}=\Varangle\left(\mathbf{p}_{W}, \mathbf{p}_{\ell_{3}}\right)$, and $\theta_{D_{b}}=\Varangle\left(\mathbf{p}_{t}, \mathbf{p}_{b}\right)$, for example,

$$
\cos \theta_{D_{1}}=\frac{\left(\mathbf{p}_{\tilde{t}}-\mathbf{p}_{t}\right) \cdot \hat{\mathbf{p}}_{\ell_{1}}}{\left|\mathbf{p}_{\tilde{t}}-\mathbf{p}_{t}\right|}
$$

with the unit momentum vector $\hat{\mathbf{p}}=\mathbf{p} /|\mathbf{p}|$. The other momenta $\mathbf{p}_{\chi_{i}^{0}}, \mathbf{p}_{\tilde{\ell}}$, and $\mathbf{p}_{W}$, follow from momentum conservation.

The Lorentz invariant phase-space element for the squark decay chain, see Eqs. (22)(4), can be decomposed into two-body phase-space elements [36, 60]

$$
\begin{aligned}
d \operatorname{Lips}\left(s_{\tilde{t}} ; p_{\nu_{\ell}}, p_{\chi_{1}^{0}},\right. & \left.p_{b}, p_{\ell_{1}}, p_{\ell_{2}}, p_{\ell_{3}}\right)=\frac{1}{(2 \pi)^{4}} \sum_{ \pm} d \operatorname{Lips}\left(s_{\tilde{t}} ; p_{t}, p_{\chi_{i}^{0}}\right) \\
& \times d s_{\chi_{i}^{0}} d \operatorname{Lips}\left(s_{\chi_{i}^{0}} ; p_{\ell_{1}}, p_{\tilde{\ell}}\right) d s_{\tilde{\ell}} d \operatorname{Lips}\left(s_{\tilde{\ell}} ; p_{\chi_{1}^{0}}, p_{\ell_{2}}\right) \\
& \times d s_{t} d \operatorname{Lips}\left(s_{t} ; p_{b}, p_{W}\right) d s_{W} d \operatorname{Lips}\left(s_{W} ; p_{\ell_{3}}, p_{\nu_{\ell}}\right)
\end{aligned}
$$

where we have to sum the two solutions $\left|\mathbf{p}_{t}^{ \pm}\right|$of the top quark momentum, see Eq. (D.27), if the decay angle $\theta_{t}$ is constrained. The different factors are

$$
\begin{aligned}
d \operatorname{Lips}\left(s_{\tilde{t}} ; p_{t}, p_{\chi_{i}^{0}}\right) & =\frac{1}{8 \pi} \frac{\left|\mathbf{p}_{t}\right|^{2}}{\left|E_{t}\right| \mathbf{p}_{\tilde{t}}\left|\cos \theta_{t}-E_{\tilde{t}}\right| \mathbf{p}_{t}||} \sin \theta_{t} d \theta_{t}, \\
d \operatorname{Lips}\left(s_{\chi_{i}^{0}} ; p_{\ell_{1}}, p_{\tilde{\ell}}\right) & =\frac{1}{2(2 \pi)^{2}} \frac{\left|\mathbf{p}_{\ell_{1}}\right|^{2}}{m_{\chi_{i}^{0}}^{2}-m_{\tilde{\ell}}^{2}} d \Omega_{1}, \\
d \operatorname{Lips}\left(s_{\tilde{\ell}} ; p_{\chi_{1}^{0}}, p_{\ell_{2}}\right) & =\frac{1}{2(2 \pi)^{2}} \frac{\left|\mathbf{p}_{\ell_{2}}\right|^{2}}{m_{\tilde{\ell}}^{2}-m_{\chi_{1}^{0}}^{2}} d \Omega_{2}, \\
d \operatorname{Lips}\left(s_{t} ; p_{b}, p_{W}\right) & =\frac{1}{2(2 \pi)^{2}} \frac{\left|\mathbf{p}_{b}\right|^{2}}{m_{t}^{2}-m_{W}^{2}} d \Omega_{b}, \\
d \operatorname{Lips}\left(s_{W} ; p_{\ell_{3}}, p_{\nu_{\ell}}\right) & =\frac{1}{2(2 \pi)^{2}} \frac{\left|\mathbf{p}_{\ell_{3}}\right|^{2}}{m_{W}^{2}} d \Omega_{3},
\end{aligned}
$$


with $s_{j}=p_{j}^{2}$ and $d \Omega_{j}=\sin \theta_{j} d \theta_{j} d \phi_{j}$. We use the narrow width approximation

$$
\int|\Delta(j)|^{2} d s_{j}=\frac{\pi}{m_{j} \Gamma_{j}}
$$

for the propagators

$$
\Delta(j)=\frac{i}{s_{j}-m_{j}^{2}+i m_{j} \Gamma_{j}},
$$

which is justified for $\Gamma_{j} / m_{j} \ll 1$, which holds in our case for particle widths $\Gamma_{j} \lesssim \mathcal{O}(1 \mathrm{GeV})$, and masses $m_{j} \approx \mathcal{O}(100 \mathrm{GeV})$. Note, however, that the naive $\mathcal{O}(\Gamma / m)$-expectation of the error can easily receive large off-shell corrections of an order of magnitude and more, in particular at threshold, or due to interferences with other resonant or non-resonant processes. For a recent discussion of these issues, see, for example, Ref. [61].

\section{E Density Matrix Formalism}

The amplitude squared for the entire stop decay chain, Eqs. (2)-(4), has been calculated in Ref. [41, by using the spin formalism of Kawasaki, Shirafuji and Tsai 62. We calculate the amplitude squared in the spin-density matrix formalism [45, 63], which allows a separation of the amplitude squared into contributions from spin correlations, spin-spin correlations and from the unpolarized part. In that way, the CP-sensitive parts of the amplitude squared can be easily separated and identified. The stop decay amplitude squared in this formalism, however for a subsequent three-body decay of the neutralino, was calculated in Ref. [43].

In the spin-density matrix formalism of Ref. [45], the amplitude squared of the stop decay chain, Eqs. (2)-(4), can be written as

$$
\begin{array}{r}
|T|^{2}=|\Delta(t)|^{2}\left|\Delta\left(\tilde{\chi}_{i}^{0}\right)\right|^{2}|\Delta(W)|^{2}|\Delta(\tilde{\ell})|^{2} \times \\
\sum_{\lambda_{i}, \lambda_{i}^{\prime}, \lambda_{t}, \lambda_{t}^{\prime}, \lambda_{k} \lambda_{k}^{\prime}} \rho_{D}(\tilde{t})_{\lambda_{i} \lambda_{i}^{\prime}}^{\lambda_{t} \lambda_{t}^{\prime}} \rho_{D_{1}}\left(\tilde{\chi}_{i}^{0}\right)^{\lambda_{i}^{\prime} \lambda_{i}} D_{2}(\tilde{\ell}) \rho_{D_{3}}(t)_{\lambda_{t}^{\prime} \lambda_{t}}^{\lambda_{k}^{\prime} \lambda_{k}} \rho_{D_{4}}(W)_{\lambda_{k} \lambda_{k}^{\prime}} .
\end{array}
$$

The amplitude squared is composed of the propagators $\Delta(j)$, Eq. (D.42), of particle $j=t, \tilde{\chi}_{i}^{0}, W$ or $\tilde{\ell}$, and the un-normalized spin density matrices $\rho_{D}(\tilde{t}), \rho_{D_{1}}\left(\tilde{\chi}_{i}^{0}\right)$, $\rho_{D_{3}}(t)$, and $\rho_{D_{4}}(W)$, with the helicity indices $\lambda_{i}, \lambda_{i}^{\prime}$ of the neutralino, the helicity indices $\lambda_{t}, \lambda_{t}^{\prime}$ of the top quark, and those of the $W$ boson, $\lambda_{k}, \lambda_{k}^{\prime}$. The decay factor for the selectron is denoted by $D_{2}(\tilde{\ell})$.

The density matrices can be expanded in terms of the Pauli matrices

$$
\begin{aligned}
\rho_{D}(\tilde{t})_{\lambda_{i} \lambda_{i}^{\prime}}^{\lambda_{t} \lambda_{t}^{\prime}}= & \delta_{\lambda_{t} \lambda_{t}^{\prime}} \delta_{\lambda_{i} \lambda_{i}^{\prime}} D+\delta_{\lambda_{i} \lambda_{i}^{\prime}} \sigma_{\lambda_{t} \lambda_{t}^{\prime}}^{a} \Sigma_{D}^{a}+\delta_{\lambda_{t} \lambda_{t}^{\prime}} \sigma_{\lambda_{i} \lambda_{i}^{\prime}}^{b} \Sigma_{D}^{b} \\
& +\sigma_{\lambda_{t} \lambda_{t}^{\prime}}^{a} \sigma_{\lambda_{i} \lambda_{i}^{\prime}}^{b} \Sigma_{D}^{a b}, \\
\rho_{D_{1}}\left(\tilde{\chi}_{i}^{0}\right)^{\lambda_{i}^{\prime} \lambda_{i}}= & \delta_{\lambda_{i} \lambda_{i}^{\prime}} D_{1}+\sigma_{\lambda_{i} \lambda_{i}^{\prime}}^{b} \Sigma_{D_{1}}^{b},
\end{aligned}
$$




$$
\begin{aligned}
\rho_{D_{3}}(t)_{\lambda_{t}^{\prime} \lambda_{t}}^{\lambda_{\lambda^{\prime} \lambda_{k}}} & =\left[\delta_{\lambda_{t}^{\prime} \lambda_{t}} D_{3}^{\mu \nu}+\sigma_{\lambda_{t}^{\prime} \lambda_{t}}^{a} \Sigma_{D_{3}}^{a \mu \nu}\right] \varepsilon_{\mu}^{\lambda_{k} *} \varepsilon_{\nu}^{\lambda_{k}^{\prime}}, \\
\rho_{D_{4}}(W)^{\lambda_{k}^{\prime} \lambda_{k}} & =D_{4}^{\rho \sigma} \varepsilon_{\rho}^{\lambda_{k}} \varepsilon_{\sigma}^{\lambda_{k}^{\prime} *},
\end{aligned}
$$

with an implicit sum over $a, b=1,2,3$.

The polarization vectors $\varepsilon_{\mu}^{\lambda_{k}}$ of the $W$ boson fulfil the completeness relation

$$
\sum_{\lambda_{k}} \varepsilon_{\mu}^{\lambda_{k}^{*} \varepsilon_{\nu}^{\lambda_{k}}}=-g_{\mu \nu}+\frac{p_{W, \mu} p_{W, \nu}}{m_{W}^{2}}
$$

with $p_{W}^{\mu} \varepsilon_{\mu}^{\lambda_{k}}=0$. Similarly the spin four-vectors $s_{t}^{a}, a=1,2,3$, for the top quark $t$, and $s_{\chi_{i}^{0}}^{b}, b=1,2,3$, for the neutralino $\tilde{\chi}_{i}^{0}$, also fulfil completeness relations

$$
\sum_{a} s_{t}^{a, \mu} s_{t}^{a, \nu}=-g^{\mu \nu}+\frac{p_{t}^{\mu} p_{t}^{\nu}}{m_{t}^{2}}, \quad \sum_{b} s_{\chi_{i}^{0}}^{b, \mu} s_{\chi_{i}^{0}}^{b, \nu}=-g^{\mu \nu}+\frac{p_{\chi_{i}^{0}}^{\mu} p_{\chi_{i}^{0}}^{\nu}}{m_{\chi_{i}^{0}}^{2}},
$$

and they form an orthonormal set

$$
s_{t}^{a} \cdot s_{t}^{b}=-\delta^{a b}, \quad s_{t}^{a} \cdot \hat{p}_{t}=0, \quad s_{\chi_{i}^{0}}^{a} \cdot s_{\chi_{i}^{0}}^{b}=-\delta^{a b}, \quad s_{\chi_{i}^{0}}^{a} \cdot \hat{p}_{\chi_{i}^{0}}=0,
$$

with the notation $\hat{p}^{\mu}=p^{\mu} / \mathrm{m}$.

The expansion coefficients of the matrices (E.44) $-($ E.46) $)$ are

$$
\begin{aligned}
& D=\frac{g^{2}}{2}\left(\left|a_{m i}^{\tilde{t}}\right|^{2}+\left|b_{m i}^{\tilde{t}}\right|^{2}\right)\left(p_{t} \cdot p_{\chi_{i}^{0}}\right)-g^{2} \operatorname{Re}\left\{a_{m i}^{\tilde{t}}\left(b_{m i}^{\tilde{t}}\right)^{*}\right\} m_{t} m_{\chi_{i}^{0}}, \\
& \Sigma_{D}^{a}=-\frac{g^{2}}{2}\left(\left|a_{m i}^{\tilde{t}}\right|^{2}-\left|b_{m i}^{\tilde{t}}\right|^{2}\right) m_{t}\left(p_{\chi_{i}^{0}} \cdot s_{t}^{a}\right), \\
& \Sigma_{D}^{b}=-\frac{g^{2}}{2}\left(\left|a_{m i}^{\tilde{t}}\right|^{2}-\left|b_{m i}^{\tilde{t}}\right|^{2}\right) m_{\chi_{i}^{0}}\left(p_{t} \cdot s_{\chi_{i}^{0}}^{b}\right) \\
& \Sigma_{D}^{a b}=\frac{g^{2}}{2}\left(\left|a_{m i}^{\tilde{t}}\right|^{2}+\left|b_{m i}^{\tilde{t}}\right|^{2}\right)\left(s_{t}^{a} \cdot s_{\chi_{i}^{0}}^{b}\right) m_{t} m_{\chi_{i}^{0}} \\
& +g^{2} \operatorname{Re}\left\{a_{m i}^{\tilde{t}}\left(b_{m i}^{\tilde{t}}\right)^{*}\right\}\left[\left(s_{t}^{a} \cdot p_{\chi_{i}^{0}}\right)\left(s_{\chi_{i}^{0}}^{b} \cdot p_{t}\right)-\left(s_{t}^{a} \cdot s_{\chi_{i}^{0}}^{b}\right)\left(p_{\chi_{i}^{0}} \cdot p_{t}\right)\right] \\
& -g^{2} \operatorname{Im}\left\{a_{m i}^{\tilde{t}}\left(b_{m i}^{\tilde{t}}\right)^{*}\right\}\left[s_{t}^{a}, p_{t}, s_{\chi_{i}^{0}}^{b}, p_{\chi_{i}^{0}}\right], \\
& D_{1}=\frac{g^{2}}{2}\left|f_{\ell i}^{R}\right|^{2}\left(m_{\chi_{i}^{0}}^{2}-m_{\tilde{\ell}}^{2}\right), \\
& \Sigma_{D_{1}}^{b}=\underset{(-)}{+} g^{2}\left|f_{\ell i}^{R}\right|^{2} m_{\chi_{i}^{0}}\left(s_{\chi_{i}^{0}}^{b} \cdot p_{\ell_{1}}\right), \\
& D_{3}{ }^{\mu \nu}=\frac{g^{2}}{2}\left[p_{b}^{\mu} p_{t}^{\nu}+p_{b}^{\nu} p_{t}^{\mu}-\left(p_{b} \cdot p_{t}\right) g^{\mu \nu}\right]_{(-)}^{+} \frac{g^{2}}{2} i \varepsilon^{\mu \alpha \nu \beta} p_{t, \alpha} p_{b, \beta}, \\
& \Sigma_{D_{3}}^{a \mu \nu}=\underset{(+)}{-} \frac{g^{2}}{2} m_{t}\left\{\left[p_{b}^{\mu} s_{t}^{a, \nu}+p_{b}^{\nu} s_{t}^{a, \mu}-\left(p_{b} \cdot s_{t}^{a}\right) g^{\mu \nu}\right]_{(-)}^{+} i \varepsilon^{\mu \alpha \nu \beta} s_{t, \alpha}^{a} p_{b, \beta}\right\}, \\
& D_{4}^{\rho \sigma}=g^{2}\left[p_{\ell_{3}}^{\rho} p_{\nu_{\ell}}^{\sigma}+p_{\ell_{3}}^{\sigma} p_{\nu_{\ell}}^{\rho}-\left(p_{\ell_{3}} \cdot p_{\nu_{\ell}}\right) g^{\rho \sigma}\right]_{(-)}^{+} g^{2} i \varepsilon^{\rho \alpha \sigma \beta} p_{\ell_{3}, \alpha} p_{\nu_{\ell}, \beta}, \\
& D_{2}(\tilde{\ell})=g^{2}\left|f_{\ell 1}^{R}\right|^{2}\left(m_{\tilde{\ell}}^{2}-m_{\chi_{1}^{0}}^{2}\right) \text {, }
\end{aligned}
$$


with the couplings as defined in Appendix C, and the short hand notation

$$
\left[p_{1}, p_{2}, p_{3}, p_{4}\right] \equiv \varepsilon_{\mu \nu \alpha \beta} p_{1}^{\mu} p_{2}^{\nu} p_{3}^{\alpha} p_{4}^{\beta} ; \quad \varepsilon_{0123}=1 .
$$

The coefficients for squark decay, Eqs. (E.51)-(E.54), agree with those given in Ref. 43. The signs in parentheses hold for the charge conjugated processes, that is $\tilde{t}_{m}^{*} \rightarrow \bar{t} \tilde{\chi}_{i}^{0}$ in Eqs. (E.52) and (E.53), $\tilde{\chi}_{i}^{0} \rightarrow \ell_{1}^{-} \tilde{\ell}_{R}^{+}$in Eq. (E.56), $\bar{t} \rightarrow \bar{b} W^{-}$in Eqs. (E.57) and (E.58), and finally $W^{-} \rightarrow \bar{\nu}_{\ell} \ell_{3}^{-}$in Eq. (E.59).

Inserting the density matrices, Eqs. (E.44)-(E.47) into Eq. (E.43), we obtain

$$
\begin{array}{r}
|T|^{2}=4|\Delta(t)|^{2}\left|\Delta\left(\tilde{\chi}_{i}^{0}\right)\right|^{2}|\Delta(W)|^{2}|\Delta(\tilde{\ell})|^{2} \times \\
{\left[\begin{array}{lll}
D & D_{1} & \left.D_{3}^{\rho \sigma}+D_{1} \Sigma_{D}^{a} \Sigma_{D_{3}}^{a \rho \sigma}+\Sigma_{D}^{b} \Sigma_{D_{1}}^{b} D_{3}^{\rho \sigma}+\Sigma_{D}^{a b} \Sigma_{D_{1}}^{b} \Sigma_{D_{3}}^{a \rho \sigma}\right] D_{4 \rho \sigma} D_{2}
\end{array}\right.}
\end{array}
$$

with an implicit sum over $a, b$. The amplitude squared $|T|^{2}$ is now composed into an unpolarized part (first summand), into the spin correlations of the top (second summand), those of the neutralino (third summand), and into the spin-spin correlations of top and neutralino (fourth summand), in Eq. (E.62).

With the completeness relation for the $W$ polarization vectors (E.48), we find

$$
\begin{aligned}
D_{3}^{\rho \sigma} D_{4 \rho \sigma} & =2 g^{4}\left(p_{t} \cdot p_{\ell_{3}}\right)\left(p_{b} \cdot p_{\nu_{\ell}}\right) \\
\Sigma_{D 3}^{a \rho \sigma} D_{4 \rho \sigma} & =-{ }_{(+)} 2 m_{t} g^{4}\left(s_{t}^{a} \cdot p_{\ell_{3}}\right)\left(p_{b} \cdot p_{\nu_{\ell}}\right),
\end{aligned}
$$

and the sign in parenthesis for the charge conjugated decay, $\bar{t} \rightarrow \bar{b} W^{-}, W^{-} \rightarrow \bar{\nu}_{\ell} \ell_{3}^{-}$. By also using the completeness relations for the top and neutralino spin vectors, Eq. (E.49), the products in Eq. (E.62) can be written as

$$
\begin{aligned}
\Sigma_{D}^{a} \Sigma_{D_{3}}^{a \rho \sigma} D_{4 \rho \sigma}= & g^{6}\left(\left|a_{m i}^{\tilde{t}}\right|^{2}-\left|b_{m i}^{\tilde{t}}\right|^{2}\right)\left(p_{b} \cdot p_{\nu_{\ell}}\right) \\
& \times\left[\left(p_{\chi_{i}^{0}} \cdot p_{t}\right)\left(p_{\ell_{3}} \cdot p_{t}\right)-m_{t}^{2}\left(p_{\chi_{i}^{0}} \cdot p_{\ell_{3}}\right)\right] \\
\Sigma_{D}^{b} \Sigma_{D_{1}}^{b}= & +\frac{g^{4}}{2}\left(\left|a_{m i}^{\tilde{t}}\right|^{2}-\left|b_{m i}^{\tilde{t}}\right|^{2}\right)\left|f_{\ell i}^{R}\right|^{2} \\
& \times\left[m_{\chi_{i}^{0}}^{2}\left(p_{t} \cdot p_{\ell_{1}}\right)-\left(p_{\chi_{i}^{0}} \cdot p_{t}\right)\left(p_{\ell_{1}} \cdot p_{\chi_{i}^{0}}\right)\right] \\
\sum_{D}^{a b} \Sigma_{D_{1}}^{b} \Sigma_{D_{3}}^{a \rho \sigma} D_{4 \rho \sigma}= & +\left({ } ^ { + } g ^ { 8 } ( | a _ { m i } ^ { \tilde { t } } | ^ { 2 } + | b _ { m i } ^ { \tilde { t } } | ^ { 2 } ) | f _ { \ell i } ^ { R } | ^ { 2 } \left[\left(p_{\chi_{i}^{0}} \cdot p_{\ell_{1}}\right)\left(p_{\chi_{i}^{0}} \cdot p_{\ell_{3}}\right) m_{t}^{2}\right.\right. \\
& +\left(p_{t} \cdot p_{\ell_{1}}\right)\left(p_{t} \cdot p_{\ell_{3}}\right) m_{\chi_{i}^{0}}^{2}-\left(p_{\ell_{1}} \cdot p_{\ell_{3}}\right) m_{\chi_{i}^{0}}^{2} m_{t}^{2} \\
& \left.-\left(p_{\chi_{i}^{0}} \cdot p_{t}\right)\left(p_{t} \cdot p_{\ell_{3}}\right)\left(p_{\chi_{i}^{0}} \cdot p_{\ell_{1}}\right)\right]\left(p_{b} \cdot p_{\nu_{\ell}}\right) \\
& +{ }_{(-)} 2 g^{8} \operatorname{Re}\left\{a_{m i}^{\tilde{t}}\left(b_{m i}^{\tilde{t}}\right)^{*}\right\}\left|f_{\ell i}^{R}\right|^{2} m_{\chi_{i}^{0}} m_{t}\left(p_{b} \cdot p_{\nu_{\ell}}\right) \\
& \times\left[\left(p_{\chi_{i}^{0}} \cdot p_{t}\right)\left(p_{\ell_{1}} \cdot p_{\ell_{3}}\right)-\left(p_{\chi_{i}^{0}} \cdot p_{\ell_{3}}\right)\left(p_{t} \cdot p_{\ell_{1}}\right)\right] \\
& +(-) \\
& 2 g^{8} \operatorname{Im}\left\{a_{m i}^{\tilde{t}}\left(b_{m i}^{\tilde{t}}\right)^{*}\right\}\left|f_{\ell i}^{R}\right|^{2} m_{\chi_{i}^{0}} m_{t}\left(p_{b} \cdot p_{\nu_{\ell}}\right)\left[p_{\ell_{3}}, p_{t}, p_{\ell_{1}}, p_{\chi_{i}^{0}}\right] .
\end{aligned}
$$


Note that $\left[p_{\ell_{3}}, p_{t}, p_{\ell_{1}}, p_{\chi_{i}^{0}}\right]=\left[p_{\tilde{t}}, p_{t}, p_{\ell_{3}}, p_{\ell_{1}}\right]$ due to momentum conservation, with the short hand notation Eq. (E.61). The signs in parentheses in Eqs. (E.66) and (E.67) hold for the charge conjugated process $\bar{t} \rightarrow \bar{b} W^{-}, W^{-} \rightarrow \bar{\nu}_{\ell} \ell_{3}^{-}$. In order to obtain the terms for the decay into a positively charged slepton, $\tilde{\chi}_{i}^{0} \rightarrow \ell_{1}^{-} \tilde{\ell}_{R}^{+}$, followed by $\tilde{\ell}_{R}^{+} \rightarrow \ell_{2}^{+} \tilde{\chi}_{1}^{0}$, one has to reverse the signs of Eqs. (E.66) and (E.67). This is due to the sign change of the term $\Sigma_{D_{1}}^{b}$, see Eq. (E.56).

For the decay into a left slepton $\tilde{\chi}_{i}^{0} \rightarrow \ell_{1}^{+} \tilde{\ell}_{L}^{-}$, Eqs. (E.55), (E.56), and (E.60) read

$$
\begin{aligned}
D_{1} & =\frac{g^{2}}{2}\left|f_{\ell i}^{L}\right|^{2}\left(m_{\chi_{i}^{0}}^{2}-m_{\tilde{\ell}}^{2}\right), \\
\Sigma_{D_{1}}^{b} & ={ }_{(+)} g^{2}\left|f_{\ell i}^{L}\right|^{2} m_{\chi_{i}^{0}}\left(s_{\chi_{i}^{0}}^{b} \cdot p_{\ell_{1}}\right), \\
D_{2}(\tilde{\ell}) & =g^{2}\left|f_{\ell 1}^{L}\right|^{2}\left(m_{\tilde{\ell}}^{2}-m_{\chi_{1}^{0}}^{2}\right),
\end{aligned}
$$

respectively. The expressions for Eqs. (E.66) and (E.67) have to be changed accordingly. The sign in parenthesis in Eq. (E.69) holds for the charge conjugated process $\tilde{\chi}_{i}^{0} \rightarrow \ell_{1}^{-} \tilde{\ell}_{L}^{+}$.

\section{F Stop Decay Widths and Asymmetry}

The partial decay width for the stop decay $\tilde{t}_{m} \rightarrow t \tilde{\chi}_{i}^{0}$ in the stop rest frame is [26]

$$
\Gamma\left(\tilde{t}_{m} \rightarrow t \tilde{\chi}_{i}^{0}\right)=\frac{\sqrt{\lambda\left(m_{\tilde{t}}^{2}, m_{t}^{2}, m_{\chi_{i}^{0}}^{2}\right)}}{4 \pi m_{\tilde{t}}^{3}} D,
$$

with the decay function $D$ given in Eqs. (E.51). For the decay $\tilde{t}_{m} \rightarrow b \tilde{\chi}_{j}^{+}$we have [26]

$$
\Gamma\left(\tilde{t}_{m} \rightarrow b \tilde{\chi}_{j}^{+}\right)=\frac{\left(m_{\tilde{t}}^{2}-m_{\chi_{j}^{+}}^{2}\right)^{2}}{16 \pi m_{\tilde{t}}^{3}} g^{2}\left(\left|k_{m j}^{\tilde{t}}\right|^{2}+\left|l_{m j}^{\tilde{t}}\right|^{2}\right),
$$

whith the approximation $m_{b}=0$. The stop-bottom-chargino couplings are [26]

$$
k_{m j}^{\tilde{t}}=\mathcal{R}_{m 1}^{\tilde{t} *} U_{j 2}^{*} Y_{b}, \quad l_{m j}^{\tilde{t}}=-\mathcal{R}_{m 1}^{\tilde{t} *} V_{j 1}+\mathcal{R}_{m 2}^{\tilde{t} *} V_{j 2} Y_{t},
$$

with the stop diagonalization matrix $\mathcal{R}^{\tilde{t}}$, Eq. (A.7), the diagonalization matrices $U$, $V$ for the chargino matrix [1,

$$
U^{*} \cdot \mathcal{M}_{\chi^{ \pm}} \cdot V^{\dagger}=\operatorname{diag}\left(m_{\chi_{1}^{ \pm}}, m_{\chi_{2}^{ \pm}}\right),
$$

and the Yukawa couplings $Y_{t}$, Eq. (C.21), and

$$
Y_{b}=\frac{m_{b}}{\sqrt{2} m_{W} \cos \beta} .
$$

The stop decay width for the entire decay chain, Eqs. (21)-(4), is given by

$$
\Gamma\left(\tilde{t} \rightarrow \nu_{\ell} \tilde{\chi}_{1}^{0} b \ell_{1} \ell_{2} \ell_{3}\right)=\frac{1}{2 m_{\tilde{t}}} \int|T|^{2} d \operatorname{Lips}\left(s_{\tilde{t}} ; p_{\nu_{\ell}}, p_{\chi_{1}^{0}}, p_{b}, p_{\ell_{1}}, p_{\ell_{2}}, p_{\ell_{3}}\right),
$$


with the phase-space element $d$ Lips given in Eq. (D.35).

We obtain an explicit expression for the asymmetry, if we insert the amplitude squared $|T|^{2}($ E.62) into Eq. (9),

$$
\mathcal{A}=\frac{\int \operatorname{Sign}(\mathcal{E}) \Sigma_{D}^{a b} \Sigma_{D_{1}}^{b} \Sigma_{D_{3}}^{a \rho \sigma} D_{4 \rho \sigma} D_{2} d \operatorname{Lips}}{\int D D_{1} D_{3}^{\rho \sigma} D_{4 \rho \sigma} D_{2} d \operatorname{Lips}},
$$

where we have already used the narrow width approximation of the propagators, see Eq. (D.41). In the numerator, only the spin-spin terms of the amplitude squared remain, since only they contain the epsilon product $\mathcal{E}$, see Eq. (8). The other terms vanish due to the phase space integration over the sign of the epsilon product, $\operatorname{Sign}(\mathcal{E})$. In the denominator, all spin and spin-spin correlation terms vanish, and only the spin-independent parts contribute. Inserting now the explicit expressions of the terms Eqs. (E.51)-(E.67) into the formula for the asymmetry, Eq. (F.77), we find

$$
\mathcal{A}=2 \eta \frac{\int \operatorname{Sign}(\mathcal{E})\left(p_{b} \cdot p_{\nu_{\ell}}\right)\left[p_{\tilde{t}}, p_{t}, p_{\ell_{3}}, p_{\ell_{1}}\right] d \operatorname{Lips}}{\left(m_{\chi_{i}^{0}}^{2}-m_{\tilde{\ell}}^{2}\right) \int\left(p_{t} \cdot p_{\ell_{3}}\right)\left(p_{b} \cdot p_{\nu_{\ell}}\right) d \operatorname{Lips}},
$$

with the coupling function

$$
\eta=\frac{\operatorname{Im}\left\{a_{m i}^{\tilde{t}}\left(b_{m i}^{\tilde{t}}\right)^{*}\right\}}{\frac{1}{2}\left(\left|a_{m i}^{\tilde{t}}\right|^{2}+\left|b_{m i}^{\tilde{t}}\right|^{2}\right) \frac{m_{\tilde{t}}^{2}-m_{\chi_{i}^{0}}^{2}-m_{t}^{2}}{2 m_{\chi_{i}^{0}} m_{t}}-\operatorname{Re}\left\{a_{m i}^{\tilde{t}}\left(b_{m i}^{\tilde{t}}\right)^{*}\right\}} .
$$

\section{G Contributions from Absorptive Phases}

The T-odd asymmetries $\mathcal{A}$, as defined in Eq. (9), are not only sensitive to the CP phases in the stop-neutralino system. Absorptive phases could also enter from finalstate interactions or finite-width effects. Although such absorptive contributions are a higher order effect, and thus expected to be small, they do not signal CP violation. However, they can be eliminated in the CP asymmetry

$$
\mathcal{A}_{\mathrm{CP}}=\frac{1}{2}(\mathcal{A}-\overline{\mathcal{A}})
$$

where $\mathcal{A}$ is the asymmetry for the stop decay,

$$
\begin{aligned}
& \tilde{t}_{m} \rightarrow t+\tilde{\chi}_{i}^{0} ; \quad \text { with } \quad t \rightarrow b+W^{+} ; \quad W^{+} \rightarrow \nu_{\ell}+\ell_{3}^{+} ; \\
& \\
& \tilde{\chi}_{i}^{0} \rightarrow \ell_{1}^{+}+\tilde{\ell}_{R}^{-} ; \quad \tilde{\ell}_{R}^{-} \rightarrow \tilde{\chi}_{1}^{0}+\ell_{2}^{-} ;
\end{aligned}
$$

and $\overline{\mathcal{A}}$ the corresponding asymmetry for the decay of the charge conjugated stop,

$$
\tilde{t}_{m}^{*} \rightarrow \bar{t}+\tilde{\chi}_{i}^{0} ; \quad \text { with } \quad \bar{t} \rightarrow \bar{b}+W^{-} ; \quad W^{-} \rightarrow \bar{\nu}_{\ell}+\ell_{3}^{-},
$$

and the same neutralino $\tilde{\chi}_{i}^{0}$ decay as in Eq. (G.82). In forming the CP asymmetry in Eq. (G.80), the absorptive phases cancel, since they do not change sign under charge conjugation. The truly $\mathrm{CP}$-violating parts of $\mathcal{A}$ however add up, since they change 
sign for the charge conjugated top decay chain. See the last term of Eq. (E.67), which is the CP-violating part of the amplitude squared.

There is one subtlety in the decay of the neutralino. It decays to equal parts into a negatively or positively charged slepton, respectively, leading to the decay chains

$$
\begin{aligned}
& \tilde{\chi}_{i}^{0} \rightarrow \ell_{1}^{+}+\tilde{\ell}_{R}^{-} ; \quad \tilde{\ell}_{R}^{-} \rightarrow \tilde{\chi}_{1}^{0}+\ell_{2}^{-} \\
& \tilde{\chi}_{i}^{0} \rightarrow \ell_{1}^{-}+\tilde{\ell}_{R}^{+} ; \quad \tilde{\ell}_{R}^{+} \rightarrow \tilde{\chi}_{1}^{0}+\ell_{2}^{+} .
\end{aligned}
$$

The two corresponding asymmetries for these two decay modes again differ in sign. This is due to a sign change in the spin correlation term of the neutralino $\Sigma_{D_{1}}^{b}$, see Eq. (E.56). One would have in principle to distinguish the near and the far leptons, $\ell_{1}$ and $\ell_{2}$ respectively, not to sum up the asymmetries to zero [41. But instead an asymmetry can be considered of a triple product which includes both leptons from the slepton decay, for example,

$$
\mathcal{T}=\left(\mathbf{p}_{\ell_{1}} \times \mathbf{p}_{\ell_{2}}\right) \cdot \mathbf{p}_{\ell_{3}} \equiv\left[\mathbf{p}_{\ell_{1}}, \mathbf{p}_{\ell_{2}}, \mathbf{p}_{\ell_{3}}\right]
$$

Then a distinction of the near and far leptons $\ell_{1}, \ell_{2}$ from the slepton decay is not needed, since there is an additional sign change due to:

$$
\left[\mathbf{p}_{\ell_{1}}, \mathbf{p}_{\ell_{2}}, \mathbf{p}_{\ell_{3}}\right]=-\left[\mathbf{p}_{\ell_{2}}, \mathbf{p}_{\ell_{1}}, \mathbf{p}_{\ell_{3}}\right]
$$

Thus one only would have to distinguish the charges of the two leptons by considering the triple product 64

$$
\mathcal{T}=\left[\mathbf{p}_{\ell^{+}}, \mathbf{p}_{\ell^{-}}, \mathbf{p}_{\ell_{3}^{+}}\right]
$$

thereby constructing the $\mathrm{CP}$ asymmetry

$$
\mathcal{A}_{\mathrm{CP}}=\frac{1}{2} \mathcal{A}\left(\left[\mathbf{p}_{\ell^{+}}, \mathbf{p}_{\ell^{-}}, \mathbf{p}_{\ell_{3}^{+}}\right]\right)-\frac{1}{2} \overline{\mathcal{A}}\left(\left[\mathbf{p}_{\ell^{+}}, \mathbf{p}_{\ell^{-}}, \mathbf{p}_{\ell_{3}^{-}}\right]\right),
$$

which is no longer sensitive to absorptive phases.

\section{References}

[1] H. E. Haber and G. L. Kane, Phys. Rept. 117, 75 (1985);

H. P. Nilles, Phys. Rept. 110 (1984) 1;

M. Drees, R. Godbole and P. Roy, Theory and phenomenology of sparticles, World Scientific, Singapur (2004);

H. Baer and X. Tata, Weak scale supersymmetry: From superfields to scattering events, Cambridge, UK: Univ. Pr. (2006).

[2] H. Goldberg, Phys. Rev. Lett. 50 (1983) 1419;

J. R. Ellis, J. S. Hagelin, D. V. Nanopoulos, K. A. Olive and M. Srednicki, Nucl. Phys. B 238 (1984) 453;

H. Pagels and J. R. Primack, Phys. Rev. Lett. 48, 223 (1982). 
[3] For an analysis of the neutralino relic density with CP phases, see, for example, G. Belanger, F. Boudjema, S. Kraml, A. Pukhov and A. Semenov, Phys. Rev. D 73, 115007 (2006) arXiv:hep-ph/0604150]; AIP Conf. Proc. 878, 46 (2006) [arXiv:hep-ph/0610110];

S. Y. Choi and Y. G. Kim, Phys. Lett. B 637, 27 (2006) arXiv:hep-ph/0602109;

J. S. Lee and S. Scopel, Phys. Rev. D 75, 075001 (2007) arXiv:hep-ph/0701221;

G. Belanger, O. Kittel, S. Kraml, H. U. Martyn and A. Pukhov, Phys. Rev. D 78, 015011 (2008) arXiv:0803.2584 [hep-ph]], arXiv:0901.4838 [hep-ph].

[4] See, for example,

H. E. Haber, Nucl. Phys. Proc. Suppl. 62, 469 (1998) arXiv:hep-ph/9709450;

T. Ibrahim and P. Nath, arXiv:hep-ph/0210251; Rev. Mod. Phys. 80, 577 (2008) arXiv:0705.2008 [hep-ph]];

J. R. Ellis, J. S. Lee and A. Pilaftsis, Phys. Rev. D 76, 115011 (2007) arXiv:0708.2079 [hep-ph]].

[5] C. Amsler et al. [Particle Data Group], Phys. Lett. B 667 (2008) 1.

[6] For a review, see for example, A. J. Buras, arXiv:hep-ph/0505175.

[7] M. B. Gavela, P. Hernandez, J. Orloff and O. Pene, Mod. Phys. Lett. A 9, 795 (1994) arXiv:hep-ph/9312215];

M. B. Gavela, P. Hernandez, J. Orloff, O. Pene and C. Quimbay, Nucl. Phys. B 430, 382 (1994) arXiv:hep-ph/9406289];

F. Csikor, Z. Fodor and J. Heitger, Phys. Rev. Lett. 82, 21 (1999) arXiv:hep-ph/9809291.

[8] For a review, see for example,

A. Riotto, arXiv:hep-ph/9807454;

W. Bernreuther, Lect. Notes Phys. 591, 237 (2002) arXiv:hep-ph/0205279.

[9] B. C. Regan, E. D. Commins, C. J. Schmidt and D. DeMille, Phys. Rev. Lett. 88, 071805 (2002).

[10] C. A. Baker et al., Phys. Rev. Lett. 97, 131801 (2006) arXiv:hep-ex/0602020.

[11] W. C. Griffith, M. D. Swallows, T. H. Loftus, M. V. Romalis, B. R. Heckel and E. N. Fortson, Phys. Rev. Lett. 102, 101601 (2009);

K. V. P. Latha, D. Angom, B. P. Das and D. Mukherjee, arXiv:0902.4790 [physics.atom-ph].

[12] Y. K. Semertzidis et al. [EDM Collaboration], AIP Conf. Proc. 698, 200 (2004) arXiv:hep-ex/0308063]; Nucl. Phys. Proc. Suppl. 131, 244 (2004) arXiv:hep-ex/0401016].

[13] J. R. Ellis, S. Ferrara and D. V. Nanopoulos, Phys. Lett. B 114, 231 (1982);

S. Abel and O. Lebedev, JHEP 0601, 133 (2006) arXiv:hep-ph/0508135. 
[14] T. Ibrahim and P. Nath, Phys. Rev. D 57 (1998) 478 [Erratum-ibid. D 58 (1998) 019901, D 60 (1999) 079903, D 60 (1999) 119901] [arXiv:hep-ph/9708456];

M. Brhlik, G. J. Good and G. L. Kane, Phys. Rev. D 59, 115004 (1999) arXiv:hep-ph/9810457;

A. Bartl, T. Gajdosik, W. Porod, P. Stockinger and H. Stremnitzer, Phys. Rev. D 60 (1999) 073003 arXiv:hep-ph/9903402;

S. Yaser Ayazi and Y. Farzan, Phys. Rev. D 74 (2006) 055008 arXiv:hep-ph/0605272;

K. A. Olive, M. Pospelov, A. Ritz and Y. Santoso, Phys. Rev. D 72, 075001 (2005) arXiv:hep-ph/0506106];

L. Mercolli and C. Smith, Nucl. Phys. B 817, 1 (2009) arXiv:0902.1949 [hep$\mathrm{ph}]$.

[15] V. D. Barger, T. Falk, T. Han, J. Jiang, T. Li and T. Plehn, Phys. Rev. D 64, 056007 (2001) arXiv:hep-ph/0101106.

[16] A. Bartl, W. Majerotto, W. Porod and D. Wyler, Phys. Rev. D 68, 053005 (2003) arXiv:hep-ph/0306050.

[17] For a recent review see, for example, J. R. Ellis, J. S. Lee and A. Pilaftsis, JHEP 0810, 049 (2008) [arXiv:0808.1819 [hep-ph]].

[18] For a connection of EDMs to ILC observables see, for example, S. Y. Choi, M. Drees and B. Gaissmaier, Phys. Rev. D 70 (2004) 014010 arXiv:hep-ph/0403054.

[19] A. Pilaftsis, Nucl. Phys. B 504 (1997) 61 arXiv:hep-ph/9702393]; Phys. Rev. D 58, 096010 (1998) arXiv:hep-ph/9803297]; Phys. Lett. B 435, 88 (1998) arXiv:hep-ph/9805373.

[20] J. F. Gunion, B. Grzadkowski, H. E. Haber and J. Kalinowski, Phys. Rev. Lett. 79 (1997) 982 arXiv:hep-ph/9704410;

B. Grzadkowski, J. F. Gunion and J. Kalinowski, Phys. Rev. D 60 (1999) 075011 arXiv:hep-ph/9902308;

D. A. Demir, Phys. Rev. D 60 (1999) 055006 arXiv:hep-ph/9901389;

S. Y. Choi, M. Drees and J. S. Lee, Phys. Lett. B 481 (2000) 57 arXiv:hep-ph/0002287;

T. Ibrahim and P. Nath, Phys. Rev. D 63, 035009 (2001) arXiv:hep-ph/0008237;

Phys. Rev. D 66, 015005 (2002) arXiv:hep-ph/0204092;

A. Pilaftsis and C. E. M. Wagner, Nucl. Phys. B 553 (1999) 3 arXiv:hep-ph/9902371;

M. S. Carena, J. R. Ellis, A. Pilaftsis and C. E. M. Wagner, Nucl. Phys. B 586 (2000) 92 arXiv:hep-ph/0003180;

M. S. Carena, J. R. Ellis, A. Pilaftsis and C. E. M. Wagner, Nucl. Phys. B 625, 345 (2002) arXiv:hep-ph/0111245;

S. Y. Choi, J. Kalinowski, Y. Liao and P. M. Zerwas, Eur. Phys. J. C 40, 555 (2005) arXiv:hep-ph/0407347; 
S. Heinemeyer, W. Hollik, H. Rzehak and G. Weiglein, Phys. Lett. B 652, 300 (2007) arXiv:0705.0746 [hep-ph]].

[21] M. Carena, J. R. Ellis, A. Pilaftsis and C. E. M. Wagner, Phys. Lett. B 495 (2000) 155 [arXiv:hep-ph/0009212].

[22] R. Barate et al. [LEP Working Group for Higgs boson searches], Phys. Lett. B 565, 61 (2003) arXiv:hep-ex/0306033;

S. Schael et al. [ALEPH Collaboration], Eur. Phys. J. C 47, 547 (2006) arXiv:hep-ex/0602042];

D. K. Ghosh and S. Moretti, Eur. Phys. J. C 42, 341 (2005) arXiv:hep-ph/0412365];

D. K. Ghosh, R. M. Godbole and D. P. Roy, Phys. Lett. B 628, 131 (2005) arXiv:hep-ph/0412193];

P. Bandyopadhyay, A. Datta, A. Datta and B. Mukhopadhyaya, Phys. Rev. D 78, 015017 (2008) arXiv:0710.3016 [hep-ph]];

A. Belyaev, Q. H. Cao, D. Nomura, K. Tobe and C. P. Yuan, Phys. Rev. Lett. 100, 061801 (2008) arXiv:hep-ph/0609079.

[23] For recent reviews and studies, see for example,

E. Accomando et al., arXiv:hep-ph/0608079;

R. M. Godbole, D. J. Miller and M. M. Muhlleitner, JHEP 0712, 031 (2007) arXiv:0708.0458 [hep-ph]];

K. E. Williams and G. Weiglein, Phys. Lett. B 660 (2008) 217 arXiv:0710.5320 [hep-ph]]; arXiv:0710.5331 [hep-ph];

H. K. Dreiner, O. Kittel and F. von der Pahlen, JHEP 0801, 017 (2008) arXiv:0711.2253 [hep-ph]];

O. Kittel and F. von der Pahlen, JHEP 0808, 030 (2008) arXiv:0806.4534 [hep-ph]];

J. S. Lee, AIP Conf. Proc. 1078, 36 (2009) [arXiv:0808.2014 [hep-ph]];

S. P. Das, A. Datta and M. Drees, AIP Conf. Proc. 1078, 223 (2009) arXiv:0809.2209 [hep-ph]];

G. Weiglein, Nucl. Phys. Proc. Suppl. 183, 149 (2008);

P. Bechtle, O. Brein, S. Heinemeyer, G. Weiglein and K. E. Williams, Comput. Phys. Commun. 181, 138 (2010) [arXiv:0811.4169 [hep-ph]];

A. Djouadi and R. M. Godbole, arXiv:0901.2030 [hep-ph];

S. Moretti, S. Munir and P. Poulose, Phys. Lett. B 649, 206 (2007) arXiv:hep-ph/0702242];

S. Hesselbach, S. Moretti, S. Munir and P. Poulose, arXiv:0903.0747 [hep-ph].

[24] S. Abdullin et al. [CMS Collaboration], J. Phys. G 28, 469 (2002) arXiv:hep-ph/9806366];

ATLAS collab., ATLAS detector and physics performance. Technical design report. Vol. 2, CERNLHCC9915;

G. Weiglein et al. [LHC/LC Study Group], Phys. Rept. 426, 47 (2006) arXiv:hep-ph/0410364. 
[25] J. Brau et al. [ILC Collaboration], arXiv:0712.1950 [physics.acc-ph];

J. A. Aguilar-Saavedra et al. [ECFA/DESY LC Physics Working Group], arXiv:hep-ph/0106315;

T. Abe et al. [American Linear Collider Working Group], arXiv:hep-ex/0106055;

K. Abe et al. [ACFA Linear Collider Working Group], arXiv:hep-ph/0109166;

J. A. Aguilar-Saavedra et al., Eur. Phys. J. C 46 (2006) 43 arXiv:hep-ph/0511344.

[26] A. Bartl, S. Hesselbach, K. Hidaka, T. Kernreiter and W. Porod, Phys. Lett. B 573, 153 (2003) arXiv:hep-ph/0307317]; Phys. Rev. D 70, 035003 (2004) arXiv:hep-ph/0311338.

[27] A. Bartl, K. Hidaka, T. Kernreiter and W. Porod, Phys. Lett. B 538 (2002) 137 arXiv:hep-ph/0204071]; Phys. Rev. D 66, 115009 (2002) arXiv:hep-ph/0207186.

[28] For the impact of CP phases in the first and second generation squarks see, for example, A. T. Alan, K. Cankocak and D. A. Demir, Phys. Rev. D 75, 095002 (2007) [Erratum-ibid. D 76, 119903 (2007)] arXiv:hep-ph/0702289].

[29] T. Gajdosik, R. M. Godbole and S. Kraml, JHEP 0409, 051 (2004) arXiv:hep-ph/0405167.

[30] S. Y. Choi and M. Drees, Phys. Lett. B 435, 356 (1998) arXiv:hep-ph/9805474;

M. Aoki and N. Oshimo, Mod. Phys. Lett. A 13, 3225 (1998) arXiv:hep-ph/9808217];

W. M. Yang and D. S. Du, Phys. Rev. D 65, 115005 (2002) arXiv:hep-ph/0202049];

T. Ibrahim and P. Nath, Phys. Rev. D 71, 055007 (2005) arXiv:hep-ph/0411272;

K. Rolbiecki and J. Kalinowski, Phys. Rev. D 76, 115006 (2007)

arXiv:0709.2994 [hep-ph]]; Acta Phys. Polon. B 39, 1585 (2008) arXiv:0804.0549 [hep-ph]]; Acta Phys. Polon. B 38, 3557 (2007) arXiv:0710.3318 [hep-ph]];

P. Osland and A. Vereshagin, Phys. Rev. D 76, 036001 (2007) arXiv:0704.2165 [hep-ph]];

M. Frank and I. Turan, Phys. Rev. D 76, 076008 (2007) arXiv:0708.0026 [hep-ph]];

O. Kittel and F. von der Pahlen, JHEP 0808, 030 (2008) arXiv:0806.4534 [hep-ph]];

H. K. Dreiner, O. Kittel and F. von der Pahlen, JHEP 0801, 017 (2008) arXiv:0711.2253 [hep-ph]];

E. Christova, H. Eberl, E. Ginina and W. Majerotto, JHEP 0702, 075 (2007) arXiv:hep-ph/0612088]; E. Christova, H. Eberl, E. Ginina and W. Majerotto, Phys. Rev. D 79, 096005 (2009) arXiv:0812.4392 [hep-ph]];

E. Christova, H. Eberl and E. Ginina, arXiv:0812.1129 [hep-ph];

H. Eberl, T. Gajdosik, W. Majerotto and B. Schrausser, Phys. Lett. B 618, 
171 (2005) arXiv:hep-ph/0502112];

W. M. Yang and D. S. Du, Phys. Rev. D 67, 055004 (2003) arXiv:hep-ph/0211453.

[31] G. Valencia, arXiv:hep-ph/9411441;

G.C. Branco, L. Lavoura, and J.P. Silva, CP violation, Oxford University Press, New York, 1999.

[32] A. Bartl, H. Fraas, O. Kittel and W. Majerotto, Phys. Rev. D 69, 035007 (2004) arXiv:hep-ph/0308141.

[33] A. Bartl, H. Fraas, T. Kernreiter and O. Kittel, Eur. Phys. J. C 33, 433 (2004) arXiv:hep-ph/0306304.

[34] For further studies with neutralino two-body decays at the ILC, see

A. Bartl, T. Kernreiter and O. Kittel, Phys. Lett. B 578, 341 (2004) arXiv:hep-ph/0309340;

S. Y. Choi, M. Drees, B. Gaissmaier and J. Song, Phys. Rev. D 69, 035008 (2004) arXiv:hep-ph/0310284;

A. Bartl, H. Fraas, O. Kittel and W. Majerotto, Eur. Phys. J. C 36, 233 (2004) arXiv:hep-ph/0402016; arXiv:hep-ph/0308143;

A. Bartl, H. Fraas, T. Kernreiter, O. Kittel and W. Majerotto, arXiv:hep-ph/0310011;

J. A. Aguilar-Saavedra, Nucl. Phys. B 697 (2004) 207 arXiv:hep-ph/0404104;

S. Y. Choi and Y. G. Kim, Phys. Rev. D 69, 015011 (2004) arXiv:hep-ph/0311037;

A. Bartl, K. Hohenwarter-Sodek, T. Kernreiter, O. Kittel and M. Terwort, JHEP 0907, 054 (2009) arXiv:0905.1782 [hep-ph]].

[35] For studies with neutralino three-body decays at the ILC, see

Y. Kizukuri and N. Oshimo, Phys. Lett. B 249 (1990) 449;

S. Y. Choi, H. S. Song and W. Y. Song, Phys. Rev. D 61, 075004 (2000) arXiv:hep-ph/9907474;

A. Bartl, H. Fraas, S. Hesselbach, K. Hohenwarter-Sodek and G. A. MoortgatPick, JHEP 0408, 038 (2004) arXiv:hep-ph/0406190];

S. Y. Choi, B. C. Chung, J. Kalinowski, Y. G. Kim and K. Rolbiecki, Eur. Phys. J. C 46, 511 (2006) arXiv:hep-ph/0504122.

[36] O. Kittel, arXiv:hep-ph/0504183.

[37] For studies with chargino two-body decays at the ILC, see

S. Y. Choi, A. Djouadi, M. Guchait, J. Kalinowski, H. S. Song and P. M. Zerwas, Eur. Phys. J. C 14, 535 (2000) arXiv:hep-ph/0002033;

A. Bartl, H. Fraas, O. Kittel and W. Majerotto, Phys. Lett. B 598, 76 (2004) arXiv:hep-ph/0406309;

O. Kittel, A. Bartl, H. Fraas and W. Majerotto, Phys. Rev. D 70, 115005 (2004) arXiv:hep-ph/0410054;

J. A. Aguilar-Saavedra, Nucl. Phys. B 717, 119 (2005) arXiv:hep-ph/0410068; 
A. Bartl, K. Hohenwarter-Sodek, T. Kernreiter, O. Kittel and M. Terwort, Nucl. Phys. B 802, 77 (2008) [arXiv:0802.3592 [hep-ph]].

[38] For studies with chargino three-body decays at the ILC, see

Y. Kizukuri and N. Oshimo, arXiv:hep-ph/9310224;

A. Bartl, H. Fraas, S. Hesselbach, K. Hohenwarter-Sodek, T. Kernreiter and G. Moortgat-Pick, Eur. Phys. J. C 51, 149 (2007) arXiv:hep-ph/0608065.

[39] G. A. Moortgat-Pick et al., Phys. Rept. 460, 131 (2008) arXiv:hep-ph/0507011;

A. Bartl, K. Hohenwarter-Sodek, T. Kernreiter and H. Rud, Eur. Phys. J. C 36 (2004) 515 arXiv:hep-ph/0403265];

A. Bartl, H. Fraas, S. Hesselbach, K. Hohenwarter-Sodek, T. Kernreiter and G. A. Moortgat-Pick, JHEP 0601, 170 (2006) arXiv:hep-ph/0510029;

S. Y. Choi, M. Drees and J. Song, JHEP 0609, 064 (2006) arXiv:hep-ph/0602131;

A. Bartl, H. Fraas, K. Hohenwarter-Sodek, T. Kernreiter, G. Moortgat-Pick and A. Wagner, Phys. Lett. B 644, 165 (2007) arXiv:hep-ph/0610431;

A. Bartl, K. Hohenwarter-Sodek, T. Kernreiter and O. Kittel, JHEP 0709, 079 (2007) arXiv:0706.3822 [hep-ph]].

[40] O. Kittel, J. Phys. Conf. Ser. 171, 012094 (2009) arXiv:0904.3241 [hep-ph]];

S. Kraml, arXiv:0710.5117 [hep-ph];

S. Hesselbach, arXiv:0709.2679 [hep-ph]; Acta Phys. Polon. B 35, 2739 (2004) arXiv:hep-ph/0410174;

A. Bartl and S. Hesselbach, arXiv:hep-ph/0410237.

[41] A. Bartl, E. Christova, K. Hohenwarter-Sodek and T. Kernreiter, Phys. Rev. D 70, 095007 (2004) arXiv:hep-ph/0409060].

[42] A. Bartl, E. Christova, K. Hohenwarter-Sodek and T. Kernreiter, JHEP 0611, 076 (2006) arXiv:hep-ph/0610234.

[43] J. Ellis, F. Moortgat, G. Moortgat-Pick, J. M. Smillie and J. Tattersall, Eur. Phys. J. C 60, 633 (2009) arXiv:0809.1607 [hep-ph]].

[44] P. Langacker, G. Paz, L. T. Wang and I. Yavin, JHEP 0707, 055 (2007) arXiv:hep-ph/0702068.

[45] H. E. Haber, arXiv:hep-ph/9405376;

H. K. Dreiner, H. E. Haber and S. P. Martin, arXiv:0812.1594 [hep-ph].

[46] A. Bartl, T. Kernreiter and W. Porod, Phys. Lett. B 538 (2002) 59 arXiv:hep-ph/0202198;

K. Kiers, A. Szynkman and D. London, Phys. Rev. D 74, 035004 (2006) arXiv:hep-ph/0605123]; Phys. Rev. D 75, 075009 (2007) arXiv:hep-ph/0701165|.

[47] J. A. Aguilar-Saavedra, Phys. Lett. B 596, 247 (2004) |arXiv:hep-ph/0403243]. 
[48] D. Atwood and A. Soni, Phys. Rev. D 45, 2405 (1992);

M. Diehl and O. Nachtmann, Z. Phys. C 62, 397 (1994);

B. Grzadkowski and J. F. Gunion, Phys. Lett. B 350, 218 (1995) arXiv:hep-ph/9501339.

[49] J. A. Aguilar-Saavedra, J. Carvalho, N. F. Castro, F. Veloso and A. Onofre, Eur. Phys. J. C 50, 519 (2007) arXiv:hep-ph/0605190]; Eur. Phys. J. C 53, 689 (2008) [arXiv:0705.3041 [hep-ph]].

[50] J. S. Lee, M. Carena, J. Ellis, A. Pilaftsis and C. E. M. Wagner, Comput. Phys. Commun. 180, 312 (2009) [arXiv:0712.2360 [hep-ph]].

[51] F. Deppisch, H. Pas, A. Redelbach, R. Ruckl and Y. Shimizu, Eur. Phys. J. C 28, 365 (2003) arXiv:hep-ph/0206122, and references therein;

F. Deppisch, H. Pas, A. Redelbach and R. Ruckl, Phys. Rev. D 73, 033004 (2006) arXiv:hep-ph/0511062, and references therein;

D. J. H. Chung, B. Garbrecht, M. J. Ramsey-Musolf, arXiv:0904.1591 [hep-ph].

[52] For recent reviews on Leptogenesis, see for example,

A. Pilaftsis, J. Phys. Conf. Ser. 171, 012017 (2009) [arXiv:0904.1182 [hep-ph]];

S. Davidson, E. Nardi and Y. Nir, Phys. Rept. 466, 105 (2008) arXiv:0802.2962 [hep-ph]];

S. Pascoli, S. T. Petcov and A. Riotto, Nucl. Phys. B 774, 1 (2007) arXiv:hep-ph/0611338;

W. Buchmuller, P. Di Bari and M. Plumacher, New J. Phys. 6, 105 (2004) arXiv:hep-ph/0406014.

[53] W. Beenakker, M. Kramer, T. Plehn, M. Spira and P. M. Zerwas, Nucl. Phys. B 515, 3 (1998) arXiv:hep-ph/9710451;

W. Hollik, M. Kollar and M. K. Trenkel, JHEP 0802, 018 (2008) arXiv:0712.0287 [hep-ph]];

M. Beccaria, G. Macorini, L. Panizzi, F. M. Renard and C. Verzegnassi, Int. J. Mod. Phys. A 23, 4779 (2008) [arXiv:0804.1252 [hep-ph]].

[54] T. Stelzer and W. F. Long, Comput. Phys. Commun. 81, 357 (1994) arXiv:hep-ph/9401258.

[55] U. Langenfeld and S. O. Moch, Phys. Lett. B 675, 210 (2009) arXiv:0901.0802 [hep-ph]];

A. Kulesza and L. Motyka, Phys. Rev. Lett. 102, 111802 (2009) arXiv:0807.2405 [hep-ph]].

[56] T. Sjostrand, S. Mrenna and P. Skands, JHEP 0605, 026 (2006) arXiv:hep-ph/0603175.

[57] W. Oller, H. Eberl and W. Majerotto, Phys. Rev. D 71 (2005) 115002 arXiv:hep-ph/0504109; Phys. Lett. B 590 (2004) 273 arXiv:hep-ph/0402134; T. Fritzsche and W. Hollik, Nucl. Phys. Proc. Suppl. 135, 102 (2004) arXiv:hep-ph/0407095. 
[58] M. Drees, W. Hollik and Q. Xu, JHEP 0702 (2007) 032 arXiv:hep-ph/0610267.

[59] A. Bartl, H. Fraas and W. Majerotto, Nucl. Phys. B 278 (1986) 1.

[60] E. Byckling, K. Kajantie, Particle Kinematics, John Wiley\& Sons, 1973.

[61] K. Hagiwara et al., Phys. Rev. D 73 (2006) 055005 arXiv:hep-ph/0512260;

D. Berdine, N. Kauer and D. Rainwater, Phys. Rev. Lett. 99, 111601 (2007) arXiv:hep-ph/0703058;

N. Kauer, Phys. Lett. B 649, 413 (2007) arXiv:hep-ph/0703077;

JHEP 0804, 055 (2008) arXiv:0708.1161 [hep-ph]];

C. F. Uhlemann and N. Kauer, Nucl. Phys. B 814, 195 (2009) arXiv:0807.4112 [hep-ph]];

M. A. Gigg and P. Richardson, arXiv:0805.3037 [hep-ph].

[62] S. Kawasaki, T. Shirafuji and S. Y. Tsai, Prog. Theor. Phys. 49 (1973) 1656.

[63] For chargino/neutralino production in the spin-density matrix formalism, see G. A. Moortgat-Pick, H. Fraas, A. Bartl and W. Majerotto, Eur. Phys. J. C 7, 113 (1999) [arXiv:hep-ph/9804306]; Eur. Phys. J. C 9, 521 (1999) [Erratumibid. C 9, 549 (1999)] [arXiv:hep-ph/9903220].

[64] J. A. Aguilar-Saavedra, private discussions. 